\title{
Instabilities and transient behaviors of a liquid film flowing down a porous inclined plane
}

\author{
Rong Liu and Qiusheng $\mathrm{Liu}^{\mathrm{a})}$ \\ Key Laboratory of Microgravity (National Microgravity Laboratory), Institute of Mechanics, \\ Chinese Academy of Sciences, Beijing 100190, China
}

(Received 26 October 2009; accepted 20 May 2010; published online 14 July 2010)

\begin{abstract}
The nonmodal linear stability of a falling film over a porous inclined plane has been investigated. The base flow is driven by gravity. We use Darcy's law to describe the flow in the porous medium. A simplified one-sided model is used to describe the fluid flow. In this model, the influence of the porous layer on the flow in the film can be identified by a parameter $\beta$. The instabilities of a falling film have traditionally been investigated by linearizing the governing equations and testing for unstable eigenvalues of the linearized problem. However, the results of eigenvalue analysis agree poorly in many cases with experiments, especially for shear flows. In the present paper, we have studied the linear stability of three-dimensional disturbances using the nonmodal stability theory. Particular attentions are paid to the transient behavior rather than the long time behavior of eigenmodes predicted by traditional normal mode analysis. The transient behaviors of the response to external excitations and the response to initial conditions are studied by examining the pseudospectral structures and the energy growth function $G(t)$. Before we study the nonmodal stability of the system, we extend the results of long-wave analysis in previous works by examining the linear stabilities for streamwise and spanwise disturbances. Results show that the critical conditions of both the surface mode and the shear mode instabilities are dependent on $\beta$ for streamwise disturbances. However, the spanwise disturbances have no unstable eigenvalue. (C) 2010 American Institute of Physics. [doi:10.1063/1.3455503]
\end{abstract}

\section{INTRODUCTION}

Flow of a falling film on an inclined plane is found in a wide range of natural processes as well as industrial applications and has been a subject of many theoretical and experimental studies for many years. Benjamin ${ }^{1}$ and $\mathrm{Yih}^{2}$ were the first to investigate the long-wave instability of a falling film on an impermeable inclined plane. Their results reveal a long surface wave that propagates downstream at about twice the basic speed at the interface. This wave is insensitive to the velocity profile in the liquid and only weakly dependent on surface tension near the critical Reynolds number. Yih's results show that a falling film may be unstable to infinitesimal periodic perturbations, and critical conditions of the Reynolds number for the onset of instability have been obtained from the solution of the Orr-Sommerfeld equation. In De Bruin's work ${ }^{3}$ this long-wave surface mode is called the "soft mode." $\mathrm{Lin}^{4}$ found a "hard mode" of instability, which is essentially a Tollmien-Schlichting shear wave in a flow with a free surface. Unfortunately, one of the boundary conditions is in error in Ref. 4. De Bruin ${ }^{3}$ pointed out the mistake in Ref. 4 and investigated the instability of corrected system in the case of zero surface tension. Floryan et al. ${ }^{5}$ studied both the soft mode and the hard mode and paid particular attention to the effects of surface tension. Their results show that the characteristics of the hard mode depend on both the angle of inclination and the surface tension.

One more complicated problem is the instability of a

a)Electronic mail: liu@imech.ac.cn. falling film over a porous inclined plate. Recently, it is of particular interest to investigate the dynamics and stability of such a problem. Pascal first ${ }^{6}$ investigated the linear stability of a Newtonian fluid flowing down an inclined porous plane. The author employed the same procedure proposed by $\mathrm{Yih}^{2}$ and the results are only valid in the long-wave limit. In his work, Darcy's law together with the Beavers-Joseph condition $^{7}$ is used to describe the flow in porous medium. However, the author made an assumption that $K / d^{2} \ll 1$ such that the flow in the porous medium can be neglected. Here $K$ is the permeability and $d$ is the thickness of the fluid film. Thus, the dynamics of the fluid layer and porous layer are decoupled. The effect of porous medium on the instability of the combined layers reduces to the influence of a slip boundary condition. This assumption was also used in Pascal's extended work ${ }^{8}$ on the problem of a power-law fluid flowing down an inclined porous plane. More recently, Sadiq and Usha ${ }^{9}$ studied the linear and weakly nonlinear stability of the surface mode of a thin Newtonian film flowing down an inclined porous plane. The authors used the same physical model with the same assumption as in Pascal's works. ${ }^{6,8}$ In the works of Pascal ${ }^{6,8}$ and Sadiq and Usha, ${ }^{9}$ these authors have not considered, however, the influence of the porous medium on the shear mode. More recently, Liu and Liu ${ }^{10}$ used a two-sided model to examine the same problem. The effects of porous medium on the surface mode and the shear mode are examined in detail. The results reveal a new instability mode, i.e., the porous mode. Comparing the results of the two-sided mode with that of the one-sided mode shows that the two models predict the qualitatively same results if 
considering the surface mode. However, the porous mode that occurs in the combined layers could not be considered in the one-sided model. Note that Pascal only studied the stability of the surface mode, so in Ref. 10, the results of shear mode could not be compared with Pascal's results of the one-sided model.

In many applied processes, preventing a transition to turbulence is a crucial problem. Different methods, such as a reduction of near-wall viscosity or by heating or cooling the walls, have been employed to control the instability behaviors. In most theoretical works, the effects of different parameters on the instabilities have been limited in the framework of the eigenvalue analysis. A careful look at previous works indicates that the eigenvalue analysis has successfully predicted the instability behaviors for some fluid systems, such as Rayleigh convection and Taylor-Couette flow. However, for some instability problems, particularly the instabilities driven by shear flow, this approach fails to match most experimental results. For example, eigenvalue analysis shows that plane Couette flow and pipe Poiseuille flow are always stable to small disturbances for all Reynolds numbers. However, experimental studies show that Couette flow becomes unstable at Reynolds number $\operatorname{Re} \approx 360$ and pipe Poiseuille flow at $\operatorname{Re} \approx 2000$. Such discrepancies lead to reexamination of the results of traditional eigenvalue analysis and a novel way, i.e., nonmodal stability theory, of describing fluid stability. For a linear problem $(d / d t) \mathbf{U}=-i \mathcal{L} \mathbf{U}$, the nonmodal analysis is based on the fact that even if all of the eigenvalues of a linear operator are distinct and lie inside the stable plane, inputs to the system may be amplified by a rather large factor if the operator $\mathcal{L}$ is non-normal, i.e., $\mathcal{L} \mathcal{L}^{*} \neq \mathcal{L}^{*} \mathcal{L}$. Here $*$ denotes the Hermit transpose. The elements of nonmodal stability theory can be referred to in Refs. 11 and 12.

Until now, most theoretical works on the linear stability of a falling film on an impermeable plane or a porous plane consider the modal stability to disturbances. Olsson and Henningson ${ }^{13}$ first examined the transient behavior of a water-table flow using non-normal stability analysis. Their results indicate that the study of the eigenvalues alone may not reveal all of the physics of the instability of a falling film, and the nonmodal effect plays an important role in determining the instability behaviors.

Motivated by previous works mentioned above, we aim to examine the nonmodal stability of a falling film on a porous plane. For our problem, all the physical eigenmodes should contribute to the transient behavior of the system. So, we will take into account both the surface mode and the shear mode which has not been studied in the works in Refs. 6,8 , and 9 . In the present paper, we focus on the effect of porous medium on the transient behaviors. This paper is organized as follows. In Sec. II, the governing equations of the physical model are established. In Sec. III, we give a brief description of the nonmodal stability theory. The numerical methods of computing pseudospectra and energy growth function $G(t)$ are presented in Sec. IV. In Sec. V, we present the results and discussions. Finally, the conclusions are given in Sec. VI.

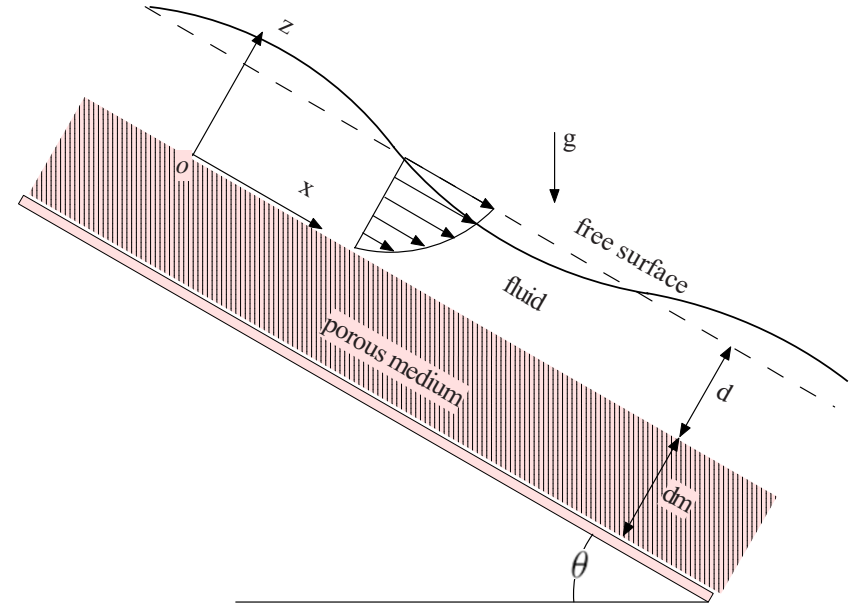

FIG. 1. (Color online) A sketch of the physical model.

\section{PROBLEM FORMULATION}

The physical model of the present study is shown in Fig. 1. A falling film of thickness $d$ on a porous plane of thickness $d_{m}$ inclined an angle $\theta$ to the horizontal direction is considered. The coordinate $x$ is parallel to basic flow and $z$ is vertical to the inclined plane. The porous media are homogeneous and sealed by an impermeable wall at $z=-d_{m}$. The combined layers are infinite in the $x$ and $y$ directions. The fluid-porous interface is located at $z=0$ and the free surface of the fluid layer is at $z=d$. The surface tension $\sigma_{0}$ of the fluid interface is assumed to be a constant. The free surface is a deformable interface described by $z=d+h(x, y, t)$; however, we assume that in base state there is no surface deflection. Thus, a fully developed, laminar flow is driven by gravity, both in the fluid and the porous layers.

\section{A. Governing equations}

In the fluid layer, the governing equations are the continuity equation and the Navier-Stokes equations

$$
\begin{aligned}
& \frac{\partial u}{\partial x}+\frac{\partial v}{\partial y}+\frac{\partial w}{\partial z}=0, \\
& \frac{\partial u}{\partial t}+u \frac{\partial u}{\partial x}+v \frac{\partial u}{\partial y}+w \frac{\partial u}{\partial z}=-\frac{1}{\rho} \frac{\partial p}{\partial x}+g \sin \theta+\nu \nabla^{2} u, \\
& \frac{\partial v}{\partial t}+u \frac{\partial v}{\partial x}+v \frac{\partial v}{\partial y}+w \frac{\partial v}{\partial z}=-\frac{1}{\rho} \frac{\partial p}{\partial y}+\nu \nabla^{2} v, \\
& \frac{\partial w}{\partial t}+u \frac{\partial w}{\partial x}+v \frac{\partial w}{\partial y}+w \frac{\partial w}{\partial z}=-\frac{1}{\rho} \frac{\partial p}{\partial z}-g \cos \theta+\nu \nabla^{2} w,
\end{aligned}
$$

where $u, v$, and $w$ are the velocity components in the $x, y$, and $z$ directions, $p$ is the pressure, $g$ is the acceleration of gravity, $\theta$ is the inclined angle, $\nu$ is the viscosity, and $\nabla^{2}$ is the Laplacian.

The flow in the porous medium is described by Darcy's model. The continuity equation and the Darcy equations are 


$$
\begin{aligned}
& \frac{\partial u_{m}}{\partial x}+\frac{\partial v_{m}}{\partial y}+\frac{\partial w_{m}}{\partial z}=0, \\
& \frac{1}{\phi} \frac{\partial u_{m}}{\partial t}=-\frac{1}{\rho} \frac{\partial p_{m}}{\partial x}-\frac{\nu}{K} u_{m}+g \sin \theta, \\
& \frac{1}{\phi} \frac{\partial v_{m}}{\partial t}=-\frac{1}{\rho} \frac{\partial p_{m}}{\partial y}-\frac{\nu}{K} v_{m}, \\
& \frac{1}{\phi} \frac{\partial w_{m}}{\partial t}=-\frac{1}{\rho} \frac{\partial p_{m}}{\partial z}-\frac{\nu}{K} w_{m}-g \cos \theta,
\end{aligned}
$$

where $u_{m}, v_{m}$, and $w_{m}$ are the Darcy velocities in the $x, y$, and $z$ directions, $p_{m}$ is the intrinsic volume average pressure, $\phi$ is the porosity, and $K$ is the permeability. For a porous medium consisting of glass spheres, $K$ is obtained by KozenyCarmen relation ${ }^{14}$

$$
K=\frac{D^{2}}{172.8} \frac{\phi^{3}}{(1-\phi)^{2}},
$$

in which $D$ is the diameter of the spheres.

At the bottom wall $z=-d_{m}$, there is no penetration

$$
w_{m}=0 \text {. }
$$

The boundary conditions at the free surface are the kinematic condition, the condition of zero shear stresses, and the condition that the normal stress balances the surface tension. These boundary conditions can be referred to in Ref. 5. At the free surface $z=d+h$, we have the kinematic condition

$$
w=\frac{\partial h}{\partial t}+u \frac{\partial h}{\partial x}+v \frac{\partial h}{\partial y},
$$

where $h$ is the interfacial deflection.

The shear stress balance condition

$$
\mathbf{t} \cdot \mathbf{T} \cdot \mathbf{n}=0
$$

and the normal stress balance condition

$$
\mathbf{n} \cdot \mathbf{T} \cdot \mathbf{n}-p=2 \sigma_{0} H,
$$

where $\sigma_{0}$ is the surface tension, $\mathbf{T}$ is the stress tensor, $\mathbf{n}$ is the unit normal to the interface, $\mathbf{t}$ is the unit tangent vector, and the mean curvature $H$ is defined by

$$
2 H=\frac{h_{x x}\left(1+h_{y}^{2}\right)+h_{y y}\left(1+h_{x}^{2}\right)-2 h_{x} h_{y} h_{x y}}{\left(1+h_{x}^{2}+h_{y}^{2}\right)^{3 / 2}},
$$

in which the subscripts $x$ and $y$ denote $\partial / \partial x$ and $\partial / \partial y$, respectively.

At the fluid-porous interface $z=0$, the boundary conditions include the Beavers-Joseph interfacial condition ${ }^{7}$

$$
\frac{\partial u}{\partial z}=\frac{\alpha_{\mathrm{BJ}}}{\sqrt{K}}\left(u-u_{m}\right), \quad \frac{\partial v}{\partial z}=\frac{\alpha_{\mathrm{BJ}}}{\sqrt{K}}\left(v-v_{m}\right),
$$

and the continuity of the normal velocities

$$
w=w_{m},
$$

and the continuity of pressure

$$
p=p_{m},
$$

where $\alpha_{\mathrm{BJ}}$ is the Beavers-Joseph coefficient.

\section{B. Base state}

As in most previous works, it is convenient to choose $u_{0}=g \sin \theta d^{2} / 2 \nu$ as the velocity scale. The scales of length, time, and pressure are $d, d / u_{0}$, and $\rho u_{0}^{2}$ for the fluid layer. Pascal $^{6}$ showed that the ratio of velocities in the fluid and the porous medium is

$$
u_{m} / u \sim\left(\frac{\sqrt{K}}{d}\right)^{2},
$$

where $\sqrt{K}$ is the characteristic length scale of the pore space in the porous medium. By assuming that $\sqrt{K}$ is much smaller than the depth of the film, i.e., $(\sqrt{K} / d)^{2} \ll 1$, the velocity in the porous medium can be neglected. Thus, the equations governing the flow in the fluid layer and in the porous layer are decoupled. In the works of Pascal ${ }^{6}$ and Sadiq and Usha, ${ }^{9}$ the one-sided model is used under the above assumption.

By neglecting the velocity in the porous layer, the nondimensional velocity of the base flow in the fluid layer is

$$
\bar{u}=-z^{2}+2 z+2 \beta,
$$

in which the parameter $\beta$ is defined as

$$
\beta=\frac{\sqrt{K}}{\alpha_{\mathrm{BJ}} d} \text {. }
$$

\section{Perturbation equations and boundary conditions}

We will consider the governing equations for infinitesimal disturbances. From this stage, $u, v, w$, and $p$ denote the disturbances of the velocities and the pressure. Neglecting the velocity in the porous layer, the linearized equations of the one-sided model come from the continuity equation and the Navier-Stokes equations, and are expressed as

$$
\begin{aligned}
& \frac{\partial u}{\partial x}+\frac{\partial v}{\partial y}+\frac{\partial w}{\partial z}=0 \\
& \frac{\partial u}{\partial t}+\bar{u} \frac{\partial u}{\partial x}+w \frac{\partial \bar{u}}{\partial z}=-\frac{\partial p}{\partial x}+\frac{1}{\operatorname{Re}} \nabla^{2} u \\
& \frac{\partial v}{\partial t}+\bar{u} \frac{\partial v}{\partial x}=-\frac{\partial p}{\partial y}+\frac{1}{\operatorname{Re}} \nabla^{2} v \\
& \frac{\partial w}{\partial t}+\bar{u} \frac{\partial w}{\partial x}=-\frac{\partial p}{\partial z}+\frac{1}{\operatorname{Re}} \nabla^{2} w
\end{aligned}
$$

The boundary conditions are (at $z=0)$

$$
u=\beta \frac{\partial u}{\partial z}, \quad v=\beta \frac{\partial v}{\partial z}, \quad w=0,
$$

at $z=1$,

$$
w=\frac{\partial h}{\partial t}+\bar{u} \frac{\partial h}{\partial x},
$$




$$
\begin{aligned}
& \frac{\partial u}{\partial z}+\frac{d^{2} \bar{u}}{d z^{2}} h+\frac{\partial w}{\partial x}=0, \\
& \frac{\partial v}{\partial z}+\frac{\partial w}{\partial y}=0, \\
& p-2 \frac{1}{\operatorname{Re}} \frac{\partial w}{\partial z}=-S_{0}\left(\frac{\partial^{2}}{\partial x^{2}}+\frac{\partial^{2}}{\partial y^{2}}\right) h-\frac{d \bar{p}}{d z} h
\end{aligned}
$$

Here the Reynolds number is defined as

$$
\operatorname{Re}=\frac{u_{0} d}{\nu}=\frac{g \sin \theta d^{3}}{2 \nu^{2}} .
$$

$S_{0}$ is the reciprocal of the Weber number

$$
S_{0}=\frac{1}{\mathrm{We}}=\frac{4 \sigma_{0} \nu^{2}}{\rho g^{2} \sin ^{2} \theta d^{5}},
$$

in which the Weber number is defined as

$$
\mathrm{We}=\frac{\rho u_{0}^{2} d}{\sigma_{0}} .
$$

In the work of Floryan et al., ${ }^{5}$ the surface tension number $\zeta$ instead of the Weber number is introduced. $\zeta$ is defined as

$$
\zeta=\left(\frac{3 \rho \sigma_{0}^{3}}{g \mu^{4}}\right)^{1 / 3} .
$$

Note that $\operatorname{Re}, S_{0}$, and $\zeta$ are not independent, and they have the relation

$$
S_{0}=\operatorname{Re}^{-5 / 3}\left(\frac{3}{2} \sin \theta\right)^{1 / 3} \zeta .
$$

\section{Velocity-vorticity formulation}

To describe the three-dimensional disturbances, another set of variables can be introduced to reduce the number of unknowns of the equations in terms of the primary variables $u, v, w$, and $p$. Let $\eta=\partial v / \partial x-\partial u / \partial y$ be the $z$ component of the vorticity. Eliminating the pressure, the Orr-Sommerfeld equation and the Squire equation can be expressed in terms of $w$ and $\eta$,

$$
\begin{aligned}
& \left(\frac{\partial}{\partial t}+\bar{u} \frac{\partial}{\partial x}\right) \nabla^{2} w-\frac{d^{2} \bar{u}}{d z^{2}} \frac{\partial w}{\partial x}=\frac{1}{\operatorname{Re}} \nabla^{2} \nabla^{2} w, \\
& \left(\frac{\partial}{\partial t}+\bar{u} \frac{\partial}{\partial x}\right) \eta=\frac{d \bar{u}}{d z} \frac{\partial w}{\partial y}+\frac{1}{\operatorname{Re}} \nabla^{2} \eta .
\end{aligned}
$$

We introduce the Fourier mode

$$
f(x, y, z, t)=f(z, t) \exp (i a x+i b y),
$$

in which $a$ and $b$ are the streamwise and the spanwise wavenumbers, respectively. The governing equations are expressed as

$$
\left(\frac{\partial}{\partial t}+i a \bar{u}\right)\left(D^{2}-k^{2}\right) w-i a D^{2} \bar{u} \cdot w=\frac{1}{\operatorname{Re}}\left(D^{2}-k^{2}\right)^{2} w,
$$

$$
\left(\frac{\partial}{\partial t}+i a \bar{u}\right) \eta=i b D \bar{u} \cdot w+\frac{1}{\operatorname{Re}}\left(D^{2}-k^{2}\right) \eta .
$$

At $z=0$, the boundary conditions are

$$
w=0, \quad D w=\beta D^{2} w, \quad \eta=\beta D \eta,
$$

at $z=1$,

$$
\begin{aligned}
& w=\left(\frac{\partial}{\partial t}+i a \bar{u}\right) h, \\
& D \eta=i b D^{2} \bar{u} \cdot h, \\
& \left(D^{2}+k^{2}\right) w=i a D^{2} \bar{u} \cdot h,
\end{aligned}
$$

$$
k^{2} S h+\left[\frac{\partial}{\partial t}+i a \bar{u}-\frac{1}{\operatorname{Re}}\left(D^{2}-3 k^{2}\right)\right] D w-i a D \bar{u} \cdot w=0,
$$

where $k^{2}=a^{2}+b^{2}, S=k^{2} S_{0}+2 \cot \theta / \mathrm{Re}$, and $D$ denotes the $z$-derivative.

The Orr-Sommerfeld equation and the Squire equation can be expressed in vector form

$$
\frac{\partial}{\partial t}\left[\begin{array}{cc}
D^{2}-k^{2} & 0 \\
0 & 1
\end{array}\right]\left[\begin{array}{l}
w \\
\eta
\end{array}\right]=\left[\begin{array}{ll}
\mathcal{L}_{\mathrm{OS}} & 0 \\
\mathcal{L}_{c} & \mathcal{L}_{\mathrm{Sq}}
\end{array}\right]\left[\begin{array}{l}
w \\
\eta
\end{array}\right] .
$$

Let $\mathcal{A}$ and $\mathcal{B}$ represent the left-hand side and the right-hand side matrix operator, respectively. We obtain the linear initial value problem

$$
\frac{\partial}{\partial t} \mathbf{u}=-i \mathcal{L} \mathbf{u}
$$

where $\mathbf{u}=(w, \eta)^{T}$ and $\mathcal{L}=i \mathcal{A}^{-1} \mathcal{B}$.

There are six independent parameters playing role in determining the instability behaviors, i.e., the two wavenumbers $a$ and $b$, the Reynolds number Re, the nondimensional surface number $\zeta$, the inclined angle $\theta$, and the parameter $\beta$. Physically speaking, $\beta$ describes the effect of the porous medium. From Eqs. (19) and (40), $\beta$ influences both the base velocity and the boundary conditions as well. From the definition of $\beta$, this parameter is related to the permeability $K$, the thickness of the liquid layer $d$, and the Beavers-Joseph coefficient $\alpha_{\mathrm{BJ}}$. The value of $\alpha_{\mathrm{BJ}}$ is proportional to the magnitude of the velocity gradient above the interface. A larger $\alpha_{\mathrm{BJ}}$ corresponds to a larger shear stress above the interface. For the base velocity, $\beta$ identifies the magnitude of the velocity slip at the interface; for perturbed state, $\beta$ identifies the influence of the shear stress.

\section{MATHEMATICAL BACKGROUND}

Before we discuss the results of the problem, it is helpful to give a brief description of the nonmodal stability theory. In nonmodal stability theory, stability is redefined in a broader sense as the response to general input variables including initial conditions, impulsive, and continuous external excitations. In a mathematical sense, an impulsive excitation is equivalent to an initial condition. In the present study, we only study responses to continuous external excitations and 
to initial conditions. The concepts of the pseudospectra, resolvent, numerical range, and optimal energy growth function, which are used to describe the nonmodal stability, can be referred to in Schmid's work. ${ }^{15}$

We begin with the responses to external excitations. Suppose a fluid system is driven by a signal of the form

$$
\mathbf{V}(x, y, z, t)=\exp (-i \omega t) \mathbf{v}(x, y, z),
$$

in which $\omega$ is the complex frequency. Then the response $\mathbf{U}(t)$ and the input signal $V$ satisfy the equation

$$
\frac{d \mathbf{U}}{d t}=-i \mathcal{L} \mathbf{U}+\exp (-i \omega t) \mathbf{v}
$$

The response is

$$
\mathbf{U}(t)=i \exp (-i \omega t) \mathbf{u}=i \exp (-i \omega t)(\omega \mathcal{I}-\mathcal{L})^{-1} \mathbf{v},
$$

where $\mathcal{I}$ is the identity matrix. The solution operator $(\omega \mathcal{I}$ $-\mathcal{L})^{-1}$ is known as the resolvent. The maximum value of amplification is equal to the norm of the resolvent

$$
\max _{\mathbf{v} \neq 0} \frac{\|\mathbf{u}\|}{\|\mathbf{v}\|}=\left\|(\omega \mathcal{I}-\mathcal{L})^{-1}\right\|,
$$

where $\|\cdot\|$ denotes a norm on $\mathrm{C}^{N}$ and max denotes maximum. An eigenvalue of $\mathcal{L}$ is a number $\omega$ such that $\left\|(\omega \mathcal{I}-\mathcal{L})^{-1}\right\|$ $\rightarrow \infty$. Generalizing this result leads naturally to the definition of $\epsilon$-pseudospectra. ${ }^{11}$

For each $\epsilon>0$, the $\epsilon$-pseudospectra $\sigma_{\epsilon}(\mathcal{L})$ of an operator $\mathcal{L}$ is the set of $\omega \in \mathbb{C}$ such that

$$
\left\|(\omega \mathcal{I}-\mathcal{L})^{-1}\right\| \geq \epsilon^{-1} .
$$

Pseudospectra can also be defined in other equivalent ways. ${ }^{11}$ For a normal operator $\mathcal{L}$, the 2 -norm of the resolvent $\mathcal{R}(\omega)$ $=\left\|(\omega \mathcal{I}-\mathcal{L})^{-1}\right\|_{2}$ is given by $\mathcal{R}(\omega)=\sup _{\sigma \in \Lambda(\mathcal{L})}|\omega-\sigma|^{-1}$, where $\Lambda(\mathcal{L})$ is the spectrum of $\mathcal{L}$ and $|\cdot|$ denotes the distance. It is apparent that when $\omega$ is far from the spectrum, the resolvent norm of a normal operator is small. However, for a nonnormal operator $\mathcal{L}$, the norm of the resolvent may be large even when $\omega$ is far from the spectrum.

Quest for maximum amplification of initial condition is of particular interest in many hydrodynamic stability problems. For a linearized fluid system,

$$
\frac{d \mathbf{u}}{d t}=-i \mathcal{L} \mathbf{u} .
$$

If the operator $\mathcal{L}$ has no time dependence, the solution has the form

$$
\mathbf{u}(t)=e^{-i \mathcal{L} t} \mathbf{u}(0) .
$$

The greatest amplification of initial condition at time $t$ is given by the growth function as

$$
G(t)=\max _{\mathbf{u}(0) \neq 0} \frac{\|\mathbf{u}(t)\|^{2}}{\|\mathbf{u}(0)\|^{2}}=\| e^{-i \mathcal{L} t \|^{2}} .
$$

When investigating the behaviors of the growth function, we are interested in two limits, one is $t \rightarrow \infty$ and the other is $t$ $\rightarrow 0$. The behaviors of the former case are determined by the instabilities of the eigenmodes. For the latter case, the initial growth rate can derived from the numerical range defined as

$$
W(\mathbf{A})=\left\{\mathbf{x}^{*} \mathbf{A} \mathbf{x}: \mathbf{x} \in \mathrm{C}^{N},\|\mathbf{x}\|=1\right\},
$$

in which $\mathbf{A} \in \mathbb{C}^{N \times N}$.

The numerical abscissa of $\mathbf{A}$ is defined as

$$
\omega(\mathbf{A})=\sup _{z \in W(\mathbf{A})} \operatorname{Im}(z)=\lim _{t \rightarrow 0} \frac{d}{d t}\left\|e^{\mathbf{A} t}\right\| .
$$

The initial growth rate of $G(t)$ corresponds to the numerical abscissa of $-i \mathcal{L}$.

\section{A. Choice of measure}

We note that both the pseudospectra and the growth function are dependent on the definition of norm. From mathematical and physical consideration, an appropriate measure of the disturbance is indispensable. The choice of measure leaves many options, and, in general, a clear and obvious choice is seldom available. ${ }^{15}$ For simple compressible flows, such as the Couette flow and the Poiseuille flow, the kinetic energy of the perturbation is a convenient and defensible choice. However, for the present problem, the effects of the free surface come into play; the definition of the measure should be reassessed. At least, all dynamic variables have to be represented for the optimization to converge. For the present problem, the energy of a disturbance consists of kinetic energy and surface potential energy. The kinetic energy density in wavenumber space can be expressed as

$$
E_{\mathrm{kin}}=\frac{1}{2 k^{2}}\left[\int_{0}^{1}\left(D w^{*} D w+k^{2} w^{*} w+\eta \eta^{*}\right) d z\right] .
$$

In wavenumber space the surface's potential energy is ${ }^{13}$

$$
E_{s}=\frac{S h h^{*}}{2} .
$$

Mathematically speaking, it is convenient to take $\|\cdot\|$ to be 2-norm and the corresponding inner product is defined as

$$
(\mathbf{u}, \mathbf{v})=\mathbf{v}^{*} \mathbf{u},
$$

where $*$ denotes the conjugate transpose. In order to measure the energy growth of a disturbance, it is convenient to define the energy norm ${ }^{13}$ as

$$
\begin{aligned}
\|\mathbf{u}\|_{E}^{2} & =E_{\mathrm{kin}}+E_{s} \\
& =\frac{1}{2 k^{2}}\left[\int_{0}^{1}\left(D w^{*} D w+k^{2} w^{*} w+\eta \eta^{*}\right) d z+k^{2} S h^{*} h\right],
\end{aligned}
$$

in which $\mathbf{u}=(w, \eta, h)$. Expressing the energy norm of $\mathbf{u}$ as $\|\mathbf{u}\|_{E}^{2}=\mathbf{u}^{*} \mathbf{E u}$, in which $\mathbf{E}$ contains the appropriate integration weight, the energy norm can be handled within the framework of the 2-norm as $\|\mathbf{u}\|_{E}^{2}=\|\mathbf{M u}\|_{2}^{2}$ with $\mathbf{M}$ arising from a Cholesky decomposition of $\mathbf{E}=\mathbf{M}^{*} \mathbf{M}^{16}$

\section{NUMERICAL METHOD}

We implement a Chebyshev collocation method to solve the pseudoeigenvalue problem. The domain of $(0,1)$ is transformed to the Chebyshev domain $(-1,1)$ by introducing $\xi$ $=2 z-1$. The variables $w$ and $\eta$ are expanded as 


$$
w=\sum_{n=0}^{N} \hat{w}_{n} T_{n}(\xi), \quad \eta=\sum_{n=0}^{N} \hat{\eta}_{n} T_{n}(\xi),
$$

in which $T_{n}$ denotes the $n$th Chebyshev polynomial. Using the Chebyshev series (61), the governing equations can be written in vector form

$$
\frac{d}{d t} \mathbf{u}=-i \mathcal{L} \mathbf{u}
$$

in which $\mathbf{u}=\left(\hat{w}_{0}, \ldots, \hat{w}_{N}, \hat{\eta}_{0}, \ldots, \hat{\eta}_{N}, h\right)$. This system of equations is required to solve for $2 N+3$ unknowns. The Chebyshev tau method and the collocation method can be used to solve eigenvalue problems. The numerical method for the general eigenvalue problem in the form $\mathbf{A x}=\omega \mathbf{B x}$ is described in Refs. 17 and 18. The detail of the implementation of the boundary conditions can be referred to in Ref. 12 . Chang et al. ${ }^{19}$ used the Chebyshev tau method to study the instability of Poiseuille flow in a fluid overlying a porous layer in the framework of the Darcy model. Liu et al. ${ }^{20}$ studied the same problem using the collocation method in the framework of the Brinkman model. Recently, Liu and Liu ${ }^{10}$ studied the stabilities of a film falling over a porous plane using the same method as that in Ref. 20. The details of the numerical method on the stability problem of flows in a fluid-porous system can be referred to in these papers.

We substitute Eq. (61) into Eq. (60), the energy norm of $\mathbf{u}$ is expressed as

$$
\|\mathbf{u}\|_{E}^{2}=\mathbf{u}^{*} \mathbf{E} \mathbf{u} .
$$

The matrix $\mathbf{E}$ is symmetric and can be decomposed in the form of $\mathbf{E}=\mathbf{M}^{*} \mathbf{M}$. The energy norm is expressed as

$$
\|\mathbf{u}\|_{E}^{2}=(\mathbf{M u}, \mathbf{M u}) .
$$

In our computation, let $\mathbf{x}=\mathbf{M u}$. Thus the energy norm of $\mathbf{u}$ is equivalent to the 2-norm of $\mathbf{x}$, and the governing equation is in the form of

$$
\frac{d}{d t} \mathbf{x}=-i \mathbf{M} \mathcal{L} \mathbf{M}^{-1} \mathbf{x} .
$$

We compute the norm of the solvent by using the relation

$$
\left\|\left(\omega \mathcal{I}-\mathcal{L}_{E}\right)^{-1}\right\|_{2}=\left[s_{\min }\left(\omega \mathcal{I}-\mathcal{L}_{E}\right)\right]^{-1},
$$

where $\mathcal{L}_{E}=\mathbf{M} \mathcal{L} \mathbf{M}^{-1}, s_{\min }\left(\omega \mathcal{I}-\mathcal{L}_{E}\right)$ denotes the smallest singular value of $\omega \mathcal{I}-\mathcal{L}_{E}$. Thus, the pseudospectra can be obtained by solving the singular value problem. It is convenient to compute the 2-norm of $\omega \mathcal{I}-\mathcal{L}_{E}$ using the singular value decomposition (SVD). The SVD function is provided in MATLAB or library software such as Linear Algebra Package (LAPACK). The use of the SVD function in MATLAB for the nonmodal stability problem can be referred to in Ref. 21 .

The growth function $G(t)$ associated with the energy norm can be computed using the method in Ref. 13. In this approach we only need the eigenvalues and the corresponding eigenvectors. The function $G(t)$ is approximated by

$$
G_{K}=\left\|F e^{-i \Lambda_{K} t} F^{-1}\right\|_{2}^{2},
$$

where $\Lambda_{K}$ is the matrix of dimension $K$ with the first $K$ eigenvalues on the diagonal. $F$ can be obtained by $A=F^{*} F$ in
TABLE I. The maximum energy growth rate $G_{\max }$ and the corresponding time $t_{\max }$ for $\beta=0$ at $\operatorname{Re}=1000$.

\begin{tabular}{llccc}
\hline \hline$a$ & $b$ & $S$ & $G_{\max }$ & $t_{\max }$ \\
\hline 0 & 2.644 & 100 & 113.1 & 53.8 \\
0 & 2.625 & 0.1 & 114.3 & 55.3 \\
0.7 & 1.3 & 0.1 & 14.8 & 12.3 \\
\hline \hline
\end{tabular}

which the components of $A$ are the inner product of the eigenvectors defined as

$$
A_{j l}=\left(q_{j}, q_{l}\right) .
$$

For the present problem, $N=80$ is enough to obtain satisfactory results for the spectra. For the nonmodal problem, all physical eigenmodes contribute to the numerical range and the energy growth function. Note that all eigenmodes are computed numerically, so highly dampened modes are inevitable. The highly dampened modes will not influence the results of the eigenvalue analysis; however, they will undermine the results of nonmodal analysis. So, numerical computation of the nonmodal problem must involve a truncation of physically unrealistic eigenmodes. In the program for plane Couette flow and Poiseuille flow, ${ }^{12}$ the modes with imaginary part less than -1.5 is truncated. In our program, we have truncated the modes with imaginary part less than -2.5 .

We have checked our computer program by comparing the maximum energy growth rate $G_{\max }$ and corresponding time $t_{\max }$ with the results of Olsson and Henningson ${ }^{13}$ for a film flowing over a rigid wall $(\beta=0)$. Our results of $\beta=0$ are listed in Table I for several typical cases. These results are quantitatively the same as that in Ref. 13.

\section{RESULTS AND DISCUSSION}

In the two-sided model, the influences of the porous layer are described by parameters $\hat{d}, \delta$, and $\alpha_{\mathrm{BJ}}{ }^{10}$ Here $\hat{d}$ is defined as $d / d_{m}$ and $\delta$ is the Darcy number defined as $\sqrt{K} / d_{m}$. However, for the simplified one-sided model, these parameters are not independent. The parameter $\beta=\delta / \alpha_{\mathrm{BJ}} \hat{d}$ identifies the effect of porous medium on the fluid layer. In general, for low permeable porous medium $\delta$ has the magnitude less than $10^{-2}$. In the experiment, ${ }^{7} \alpha_{\mathrm{BJ}}$ is in the range of $0.1-1$. We assume that the thickness of the liquid layer have the same magnitude with that of the porous layer such that $\hat{d}-1$. So, the magnitude of $\beta$ is in the range of $0-0.1$ and $(\sqrt{K} / d)^{2}$ is in the range of $0-10^{-4}$. In this case, the velocity in the porous medium can be safely neglected. The condition of $\beta \rightarrow 0$ and $(\sqrt{K} / d)^{2} \rightarrow 0$ corresponds to the limit of nonpermeable case. In the present paper, $\beta$ is limited in the range of $0-0.3$, which is the same with the range in the work of Pascal. ${ }^{6}$ In the work of Sadiq and Usha, ${ }^{9} \beta$ is in the range of $0-0.4$. We note that the values of $\beta$ which we use in the present paper are not intended to be physically realistic; rather, they allow us to characterize the influence of this parameter on the instability of the system. 


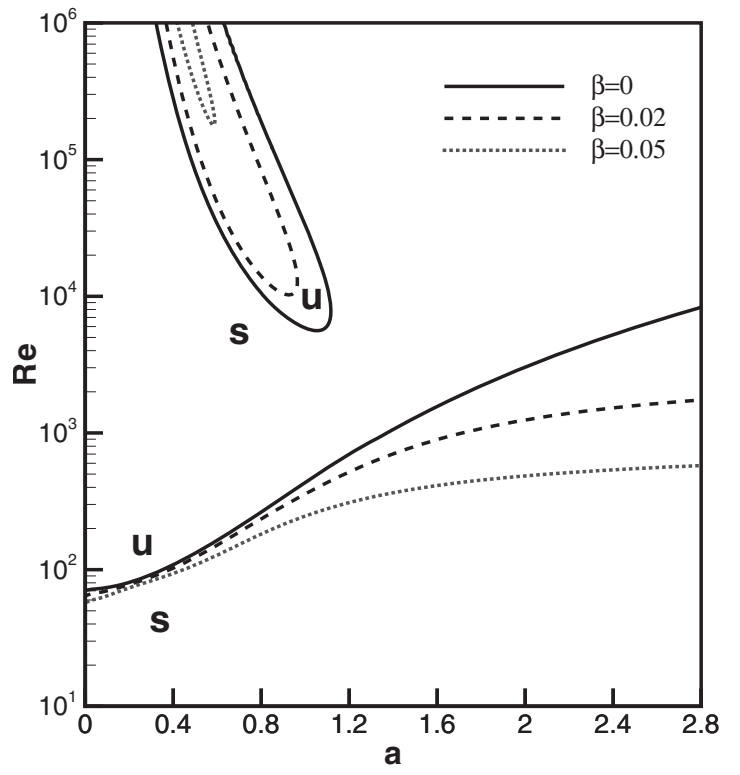

FIG. 2. Marginal curves of the Reynolds number vs the streamwise wavenumber $a$ for two-dimensional disturbances with several typical values of $\beta=0,0.02,0.05$. The other parameters are $\theta=1^{\circ}, b=0$, and $\zeta=0$.

\section{A. Eigenvalues analysis}

Floryan et al. ${ }^{5}$ studied the effect of the surface tension number $\zeta$ on the stability to two-dimensional disturbances for a falling film on an impermeable wall. Pascal ${ }^{6}$ studied the effect of $\beta$ on the stability of surface mode in the framework of a long-wave approximation. In the work of Pascal, ${ }^{6}$ the author only considered the stability of the surface mode in the framework of long-wave analysis. Note that these results are only valid in the long-wave range. In order to know the influences of the porous medium on the stability of the shear mode at all wavenumbers, we re-examine the problem by solving the controlling equations numerically. Now we aim to examine the influence of $\beta$ on the marginal curves of both the surface mode and the shear mode. As shown in previous works, for large inclined angles the surface mode is more unstable than the shear mode. In the present work, we are interested in both the surface mode and the shear mode, so we follow Floryan's work ${ }^{5}$ and limit our analysis on the case in which the plane is slightly inclined. We studied the stability for two typical cases of $\theta=1^{\circ}$ and $0.5^{\prime}$.

In this section, we begin by examining the stability to two-dimensional streamwise disturbances with the spanwise wavenumber $b=0$. In Fig. 2, we present the marginal curves for different $\beta$ at $\theta=1^{\circ}$. In this figure, the marginal curve of each $\beta$ displays a bimodal structure, i.e., the surface mode and the shear mode. The upper branches of lobe shape are of the shear mode, and the lower branches are of the surface mode. Each curve divides the $a$-Re plane into stable and unstable regions. The inner region of a lobe shape curve is unstable for the shear mode, and the upper region of the marginal curve of the surface mode is unstable region. We use "s"/"u" to denote the stable/unstable region, respectively. In Fig. 2, we label the stable/unstable of surface mode and shear mode for $\beta=0$. For $\beta=0$, the problem reduces to the case of a falling film on an impermeable plane. For each $\beta$,

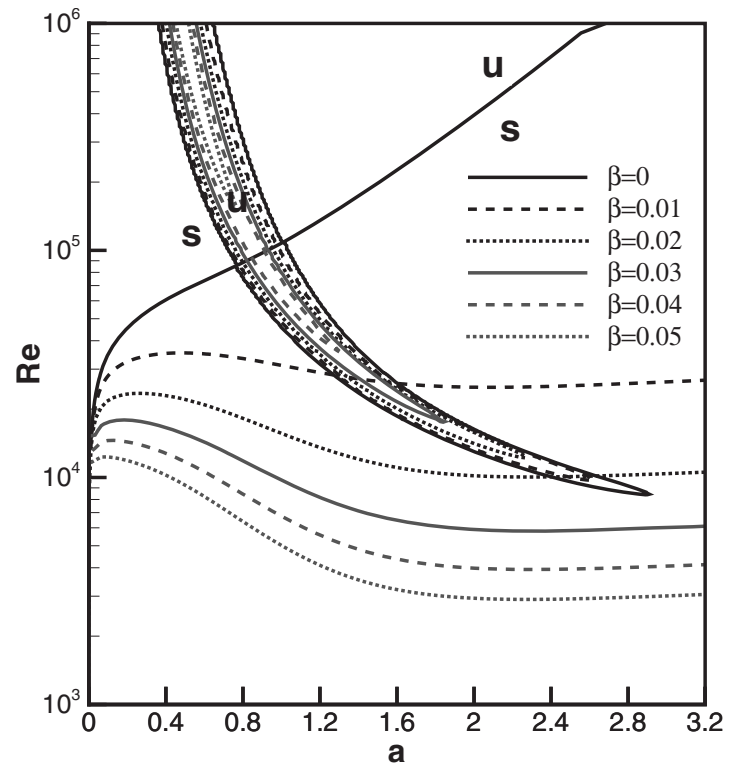

FIG. 3. Marginal curves of the Reynolds number vs the streamwise wavenumber $a$ for two-dimensional disturbances with several typical values of $\beta=0,0.01,0.02,0.03,0.04,0.05$. The other parameters are $\theta=0.5^{\prime}, b=0$, and $\zeta=0$.

the value in the surface branch monotonically increases with the wavenumber $a$, and the critical wavenumber is $a_{c}=0$. This means that at the onset of instability, the long-wave surface mode is dominant. The curves of the surface mode show that the critical value of this mode decreases slightly with the increase of $\beta$ in the long-wave region. It is apparent that with the increase of $\beta$ the marginal values in the short wave region decrease much more than that in the long-wave region.

In Fig. 2, we found that the shear mode is very sensitive to $\beta$. A slight increase of $\beta$ from 0 to 0.02 , which only results in a velocity slip about $4 \%$ of $u_{0}$ at the fluid-porous interface, will significantly stabilize the shear mode by raising the critical Reynolds number from about 6000 to 10000 . As $\beta$ increases to 0.05 , the marginal curve of the shear branch significantly shrinks in the $a$-Re plane and the critical value becomes much higher than that of $\beta=0.02$. As $\beta$ increases further, the shear mode disappears in Fig. 2.

In Fig. 3, the marginal curves are represented for a rather small inclined angle of $\theta=0.5^{\prime}$. As shown in this figure, each marginal curve also displays a bimodal structure. The branches of the long horn shape are of the shear mode and the lower branches are of the surface mode. In Fig. 3, we label the stable and the unstable regions of these two modes for $\beta=0$. For $\beta=0$, the critical Reynolds number is $\operatorname{Re}_{c}$ $=8594.4$ and the critical wavenumber is $a_{c}=0$. The curves of surface mode show that the critical value of the surface mode is insensitive to $\beta$. In comparison with that of $\theta=1^{\circ}$, at $a$ $=0$ the Reynolds numbers of surface mode of $\theta=0.5^{\prime}$ are much higher. For $\beta=0$, the values of the surface mode are much lower in the long-wave region than that in the short wave region. Being similar to the case of $\theta=1^{\circ}$, with the increase of $\beta$ the surface mode apparently becomes more unstable. We note that with the increase of $\beta$, a relative minimum enters the right part of the surface branch. For $\beta$ 

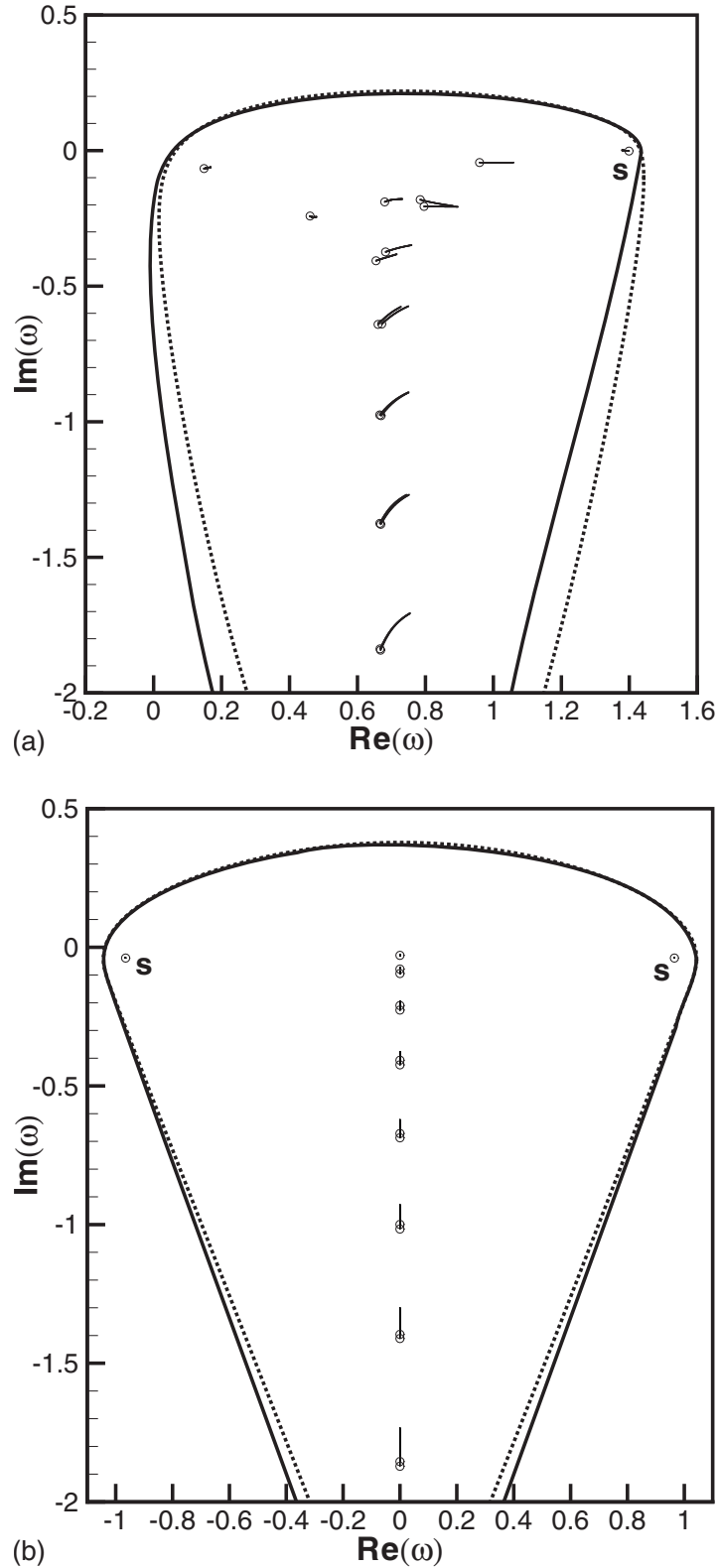

FIG. 4. The trajectories of spectra as $\beta$ increases from 0 to 0.05 (a) for streamwise disturbance of $a=1, b=0$ and (b) for spanwise disturbance of $a=0, b=1$. The solid line and the dotted line denote the numerical range of $\beta=0$ and 0.05 , respectively. The circles denote the spectra of $\beta=0$. The other parameters are $\theta=1^{\circ}, \zeta=0$, and $\operatorname{Re}=300$.

$=0.02$, the minimum in the vicinity of $a=2.0$ is approximately equal to the Reynolds number at $a=0$. As $\beta$ increases further, the most unstable disturbance occurs at $a \approx 2$. This result predicts the fact that with the increase of $\beta$, the instability switches from the long-wave mode to the short-wave mode.

In Fig. 3, the influence of $\beta$ on the shear mode for $\theta$ $=0.5^{\prime}$ is similar to that for $\theta=1^{\circ}$. As $\beta$ increases from 0 to 0.05 , the critical value of the shear mode increases from about $10^{4}$ to more than $10^{5}$. As shown in Figs. 2 and 3, the increase of $\beta$ significantly stabilizes the shear mode.

As discussed in Sec. I, the one-sided and the two-sided models predict similar results when considering the surface mode. However, the validity of the one-sided model for the
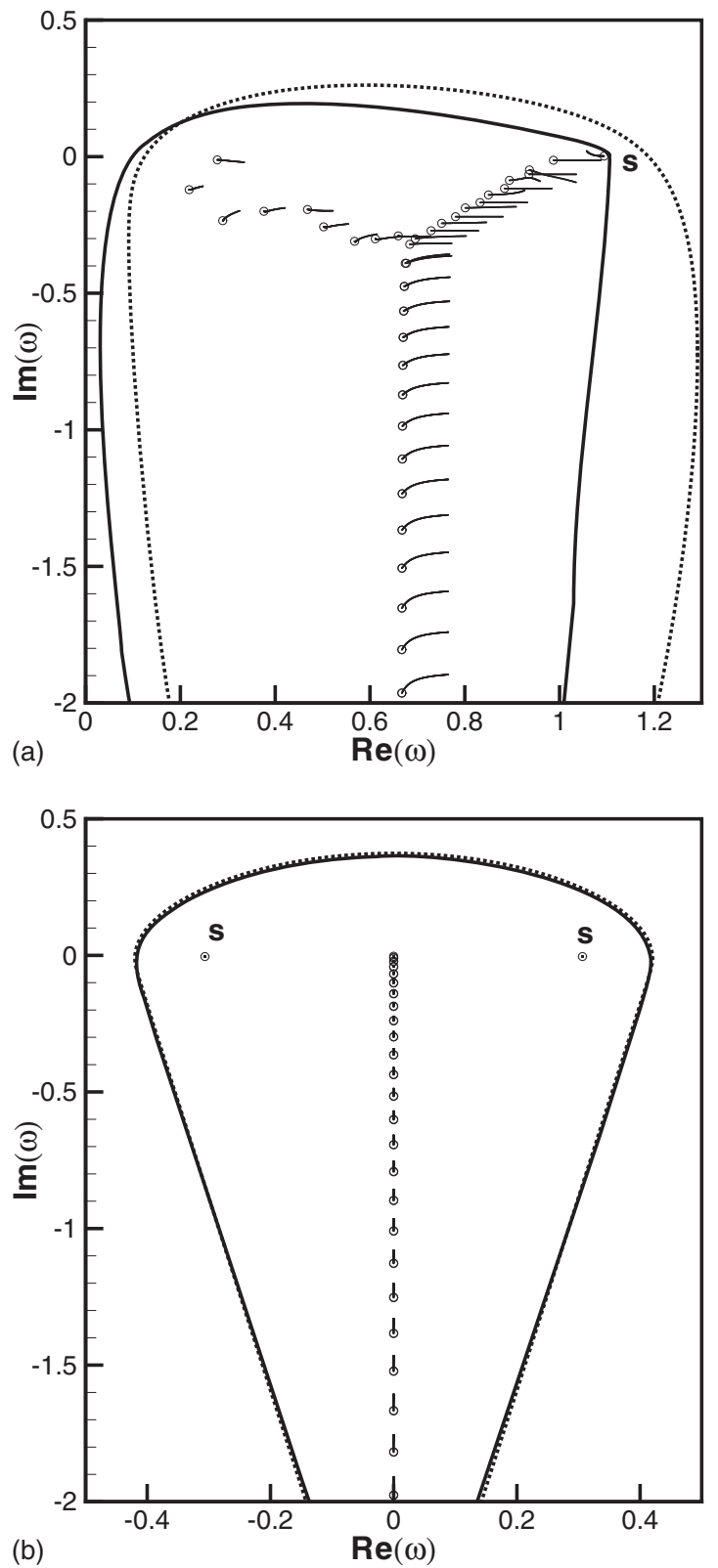

FIG. 5. The trajectories of spectra as $\beta$ increases from 0 to 0.05 (a) for streamwise disturbance of $a=1, b=0$, and (b) for spanwise disturbance of $a=0, b=1$. The solid line and the dotted line denote the numerical range of $\beta=0$ and 0.05 , respectively. The circles denote the spectra of $\beta=0$. The other parameters are $\theta=1^{\circ}, \zeta=0$, and $\operatorname{Re}=3000$.

shear mode has not been justified. Here, we will compare the results of one-sided mode with that of the two-sided mode for the shear mode. Liu and $\mathrm{Liu}^{10}$ investigated the effect of porous medium on the stability of the shear mode in the framework of the two-sided model. In Ref. 10, the marginal curves for various $\alpha_{\mathrm{BJ}}$ with $\delta=0.001, \theta=1^{\circ}$, and $\hat{d}=1$ are presented. The results show that with the increase of $\alpha_{\mathrm{BJ}}$, the shear mode becomes more unstable. Note that $\beta=\delta / \alpha_{\mathrm{BJ}} \hat{d}$. The marginal curves in Fig. 2 for the one-sided model show that with the increase of $\beta$, the shear mode becomes more stable. This means that for shear mode the results of the one-sided model are consistent with that of the two-sided model. In Ref. 10, the critical value of the shear mode of the 

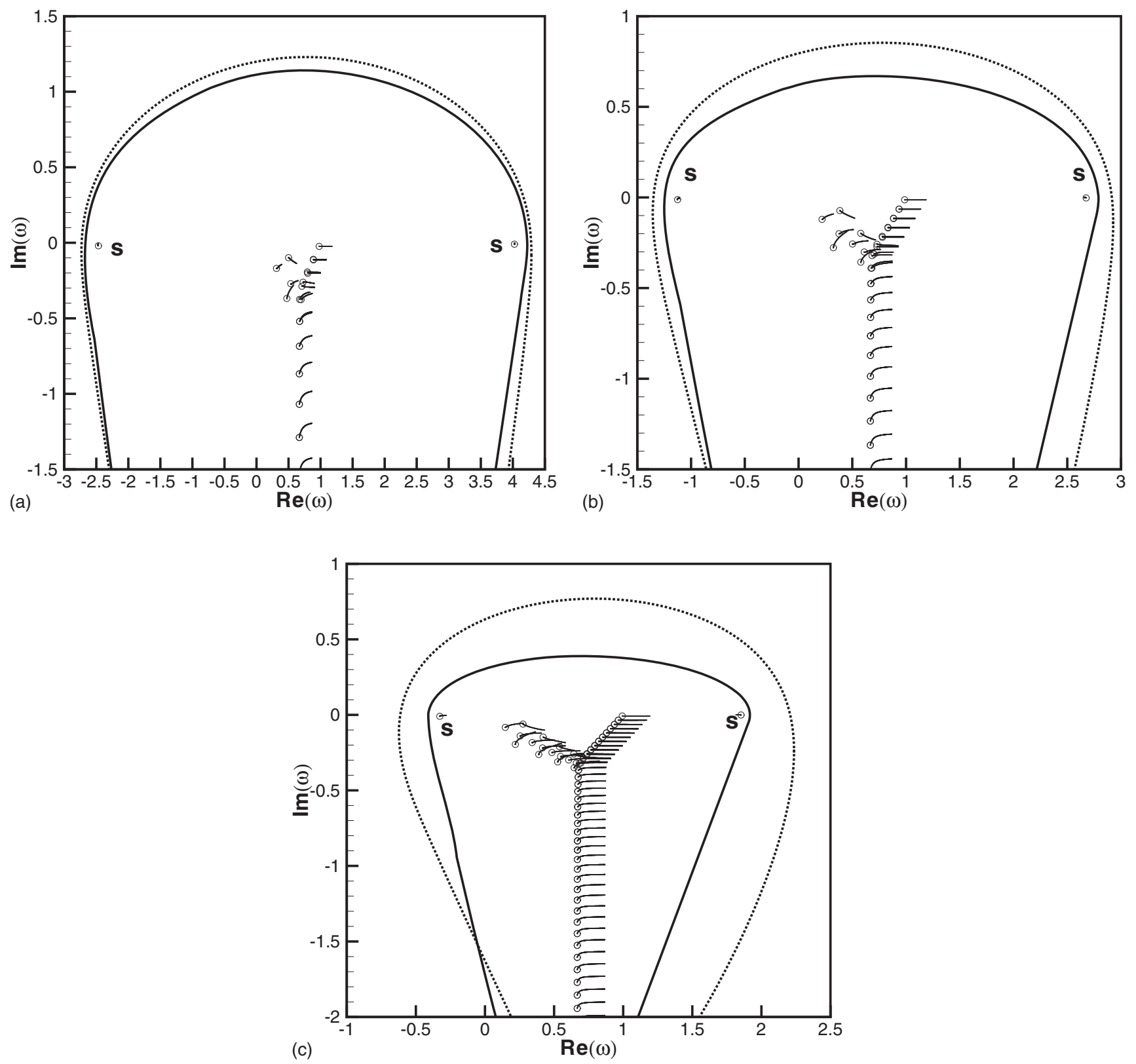

FIG. 6. The trajectories of spectra for streamwise disturbances of $a=1, b=0$ as $\beta$ increases from 0 to 0.05 at various Reynolds numbers: (a) Re $=1000$, (b) $\operatorname{Re}=3000$, and (c) $\operatorname{Re}=10000$. The circles denote the spectra of $\beta=0$. The solid line and the dotted line denote the numerical range of $\beta=0$ and 0.05 , respectively. The other parameters are $\theta=0.5^{\prime}$ and $\zeta=0$.

two-sided model is about 10000 for $\delta=0.001, \alpha_{\mathrm{BJ}}=0.05, \hat{d}$ $=1$, and $\theta=1^{\circ}$. The parameters correspond to the case of $\beta$ $=0.02$ for the one-sided model. In Fig. 2, the critical value of the shear mode of $\beta=0.02$ is about 10000 . This shows that the one-sided and two-sided models predict quantitatively the same results. As a result, the one-sided model is valid for the shear mode as well.

\section{B. Nonmodal analysis}

Because the linear operator of the problem is nonnormal, so all physical eigenmodes play roles in influencing the transient growth of the disturbances. Before we study the nonmodal instability, understanding the effects of $\beta$ on the behaviors of the eigenvalues is essential. Olsson and
Henningson ${ }^{13}$ presented the spectra, pseudospectra, and the numerical range for $S=0.1$ and $\mathrm{Re}=1000$. In the absence of surface tension, the case of $S=0.1$ and $R e=1000$ corresponds to $\theta=1.15^{\circ}$. As discussed in Sec. IV, the marginal curves of $\theta=0.5^{\prime}$ presents significantly different characters to that of $\theta=1^{\circ}$. So, following question should be asked. Is the structure of spectra of a very small $\theta$ qualitatively different to that of a larger $\theta$ ?

In the framework of nonmodal theory, we are interested in two problems, i.e., the response to external excitation and the transient energy growth of initial condition. For the former, the norm of resolvent denotes the maximum amplification; for the latter, the growth function $G(t)$ identifies the optimal growth of energy at $t$. Knowing all the information 

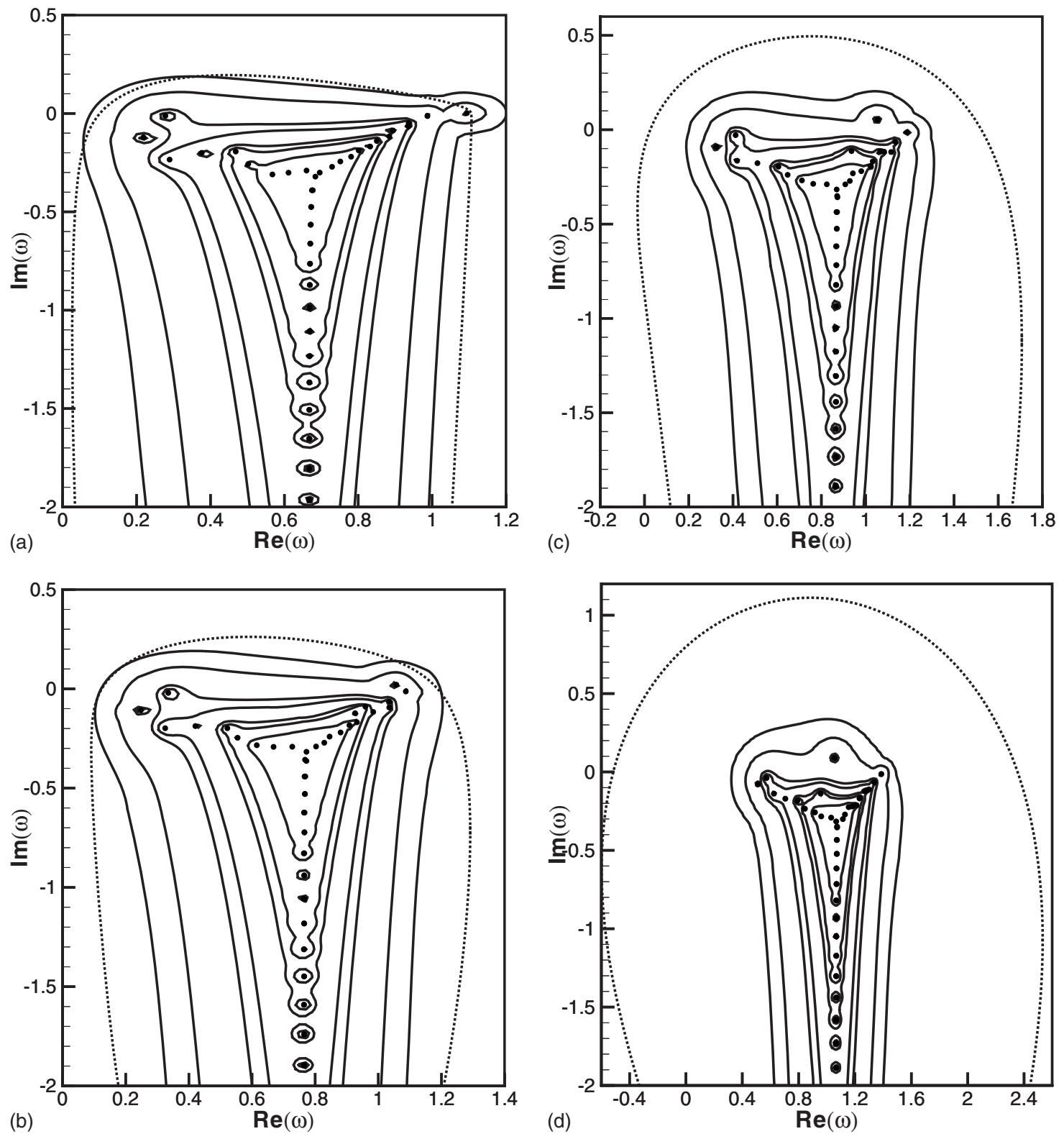

FIG. 7. Pseudospectra (solid lines) and the numerical range (dotted line) for streamwise disturbance of $a=1, b=0$. The contours of $\epsilon$ are from outer to inner $\epsilon=0.1,0.05,0.01,0.005,0.001,0.0005,0.0001$. $\epsilon$ equals the inverse of the resolvent norm. (a) $\beta=0$, (b) $\beta=0.05$, (c) $\beta=0.1$, and (d) $\beta=0.2$. The other parameters are $\theta=1^{\circ}, \zeta=0$, and $\operatorname{Re}=3000$.

of eigenvalues and eigenvectors, we can determine the behavior of the transient growth. So, examination of the behaviors of eigenvalues for different parameters is essential to understand how these parameters influence the nonmodal stability of the flow.

\section{Influences of $\beta$ on the structure of eigenvalues and the numerical ranges}

In Figs. 2 and 3, the marginal curves of $\theta=1^{\circ}$ and $0.5^{\prime}$ present different structures. Now, we will study the effect of porous medium on the structures of spectra for $\theta=1^{\circ}$ and $0.5^{\prime}$. In order to understand the influence of $\beta$ on the eigenvalues, we will display the trajectory of eigenvalues for various $\beta$ by fixing other parameters such as the Reynolds number, the wavenumbers, and the inclined angle. The Reynolds number plays an important role in determining the structure of spectra. When investigating the influence of $\beta$, we will present the structures of spectra for different values of the Reynolds number.

We begin with the structures of spectra for $\theta=1^{\circ}$. In Fig. 4 , the trajectories of the spectra as $\beta$ changes from 0 to 0.05 and numerical range of $\beta=0$ and 0.05 are presented for $\theta$ $=1^{\circ}$ at a low Reynolds number $\operatorname{Re}=300$. The imaginary part of $\omega$ presents the growth rate of an eigenmode, and the real part presents the frequency. As shown in Fig. 4(a), for the streamwise disturbance with $a=1, b=0$, the spectra display a "Y"-shaped structure, which is the same as that of Poiseuille flow, and a surface mode labeled "s" enters the region near the right part of the $\mathrm{Y}$ branch. In this figure, it is apparent that the eigenvalues in the middle branch move toward the unstable plane, and the imaginary part of $\omega$ of other modes changes little as $\beta$ increases from 0 to 0.05 . This means that 

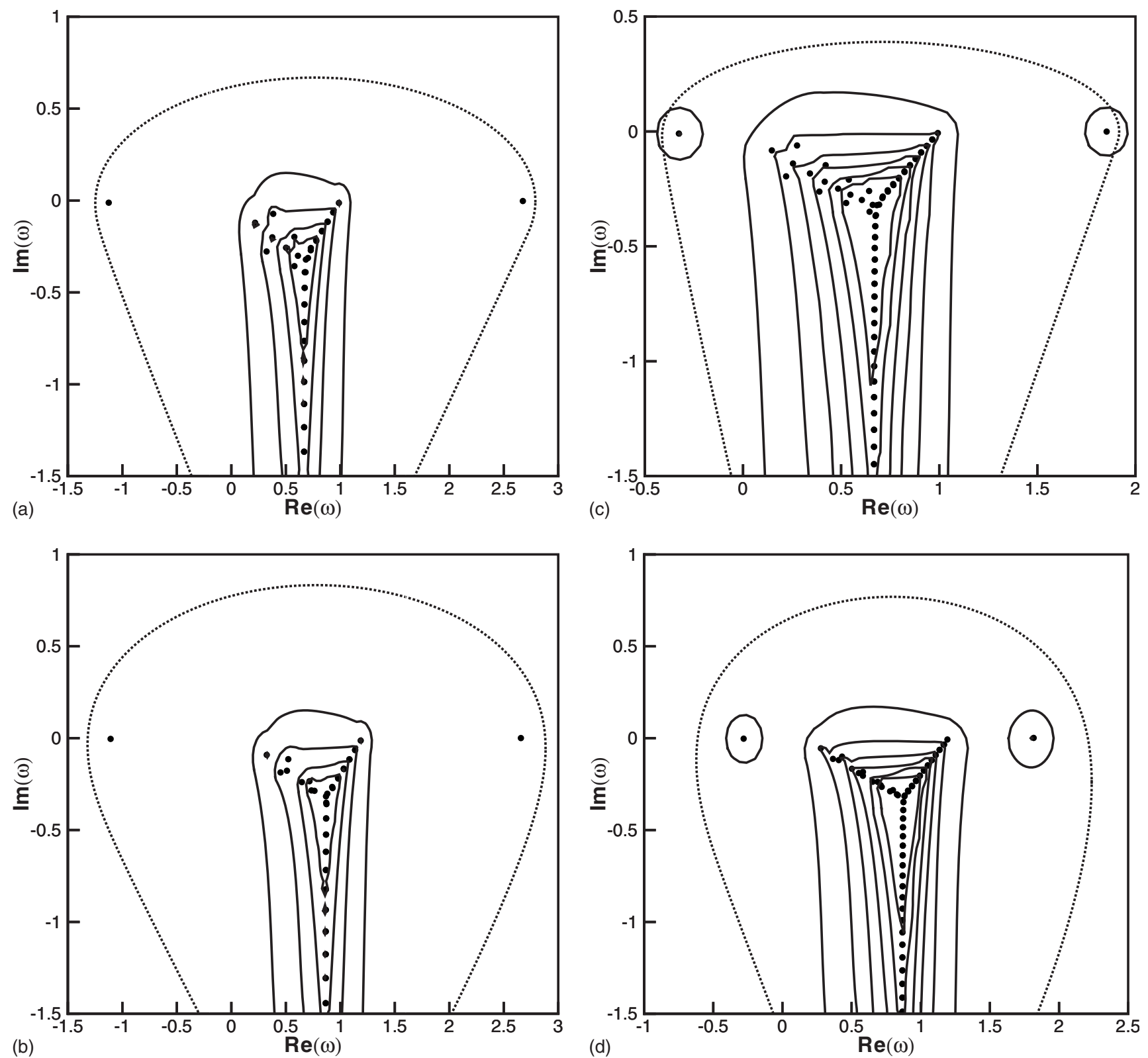

FIG. 8. Pseudospectra (solid lines), and the numerical range (dotted line) for streamwise disturbance of $a=1, b=0$. The contours of $\epsilon$ are from outer to inner $\epsilon=0.1,0.01,0.001,0.0001$ for (a) and (b) and $\epsilon=0.1,0.01,0.001,0.0001,0.00001,0.000001$ for (c) and (d). $\epsilon$ equals the inverse of the resolvent norm. (a) $\beta=0, \operatorname{Re}=3000$, (b) $\beta=0.1, \operatorname{Re}=3000$, (c) $\beta=0, \operatorname{Re}=10000$, and (d) $\beta=0.1, \operatorname{Re}=10000$. The other parameters are $\theta=0.5^{\prime}$ and $\zeta=0$.

the increase of $\beta$ significantly decreases the stabilities of the eigenvalues located in the middle branch. However, the stability of the surface mode and the eigenvalues in the left and right branches are insensitive to $\beta$. For $\operatorname{Re}=300$, the increase of $\beta$ significantly increases the frequencies of the eigenvalues in the middle and right branches. However, the frequencies of the surface mode and the eigenvalues in the left branches are insensitive to $\beta$.

The trajectories of eigenvalues of spanwise disturbances are presented in Fig. 4(b) for $\mathrm{Re}=300$ as $\beta$ increases from 0 to 0.05 . In this figure, the spectra contain Squire modes along the vertical branch and two symmetrical surface modes with the same growth rates and propagating in the opposite directions at the same magnitude of velocity. In this figure, it seems that the increase of $\beta$ only influences the stability of highly dampened eigenvalues, and the two eigenvalues of surface mode and the eigenvalues in the vicinity of $\operatorname{Im}(\omega)$ $=0$ are insensitive to $\beta$.

Figures 5(a) and 5(b) show the trajectories of spectra as $\beta$ increases from 0 to 0.05 and numerical ranges of $\beta=0$ and 0.05 for streamwise and spanwise disturbances at a higher Reynolds number $\operatorname{Re}=3000$ with the inclined angle $\theta=1^{\circ}$. The structures of spectra in Figs. 5(a) and 5(b) are qualitatively similar to that in Figs. 4(a) and 4(b). Comparing Fig. 5(a) with Fig. 4(a), it is shown that the discrete eigenvalues in the Y-shaped branches have a tendency of being more continuous with the growth of the Reynolds number. Comparing Figs. 5(b) and 4(b), it is also found that with the growth of Re, the Squire modes become more continuous. In Figs. 5(a) and 5(b), the influences of $\beta$ on the trajectories of 

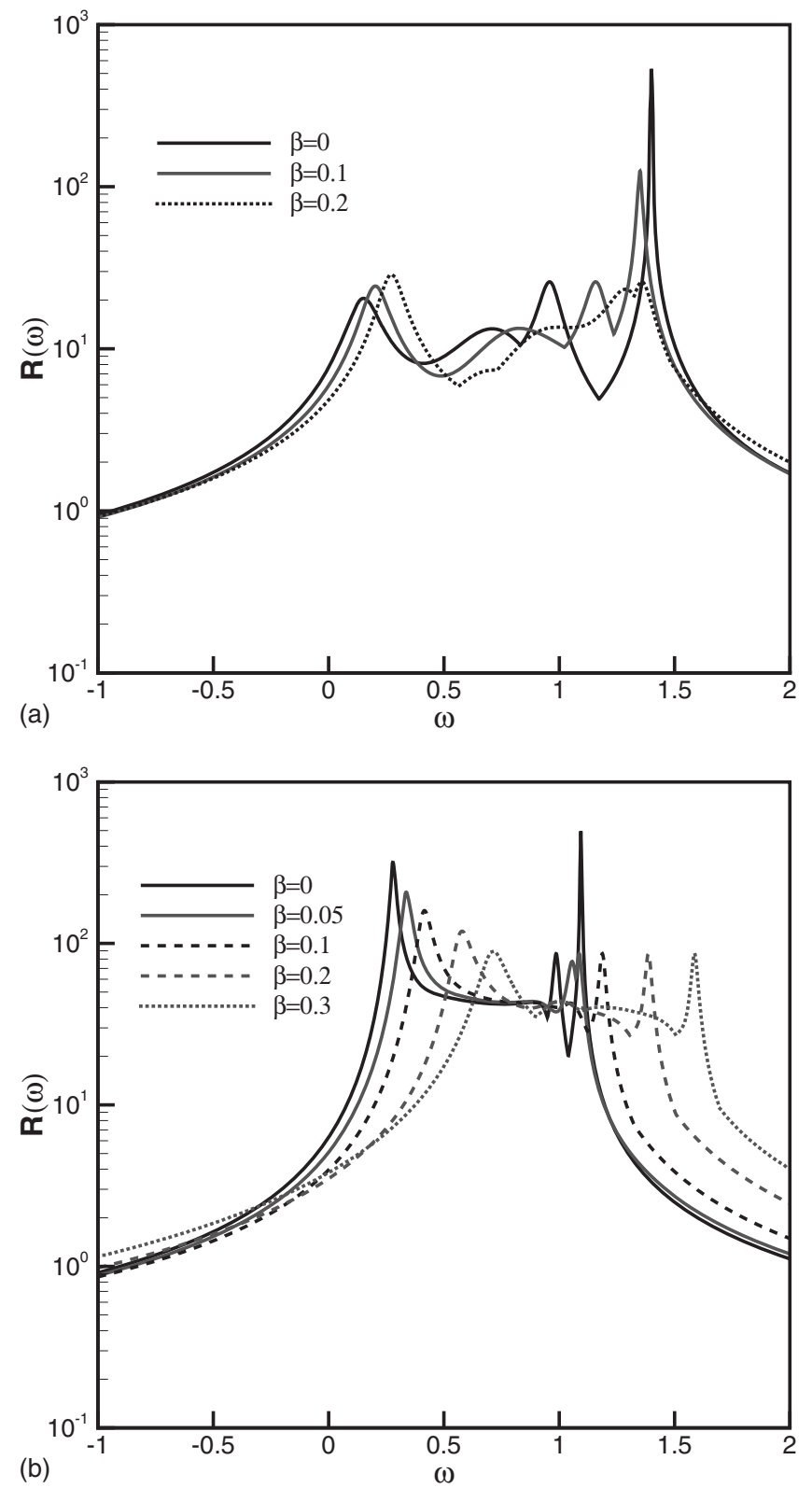

FIG. 9. Curves of the maximum response $R(\omega)$ to external excitation vs the frequency for the streamwise disturbance of $a=1, b=0$ with various $\beta$. (a) $\operatorname{Re}=300$ and (b) $\operatorname{Re}=3000$. The other parameters are $\theta=1^{\circ}$ and $\zeta=0$.

eigenvalues are qualitatively the same with that at $\operatorname{Re}=300$ in Figs. 4(a) and 4(b). In Fig. 5(a), it is observed that with the increase of $\beta$, the surface mode becomes less stable or more unstable. This result is consistent with the fact in Fig. 2 that the surface mode becomes more unstable with the increase of $\beta$.

The numerical abscissa identifies the initial energy growth rate. In Figs. 4(a) and 4(b) at $\mathrm{Re}=300$, the increase of $\beta$ has little influence on the numerical range and the numerical abscissa for both the streamwise and the spanwise disturbances. In Fig. 5(a), it is found that the numerical abscissa significantly increases as $\beta$ increases from 0 to 0.05 . However, in Fig. 5(b), the increase of $\beta$ has little influence on the numerical range and numerical abscissa. These results indicate that for $\theta=1^{\circ}$ the initial growth rate of $G(t)$ is insensitive to $\beta$ at a low Reynolds number for streamwise distur-
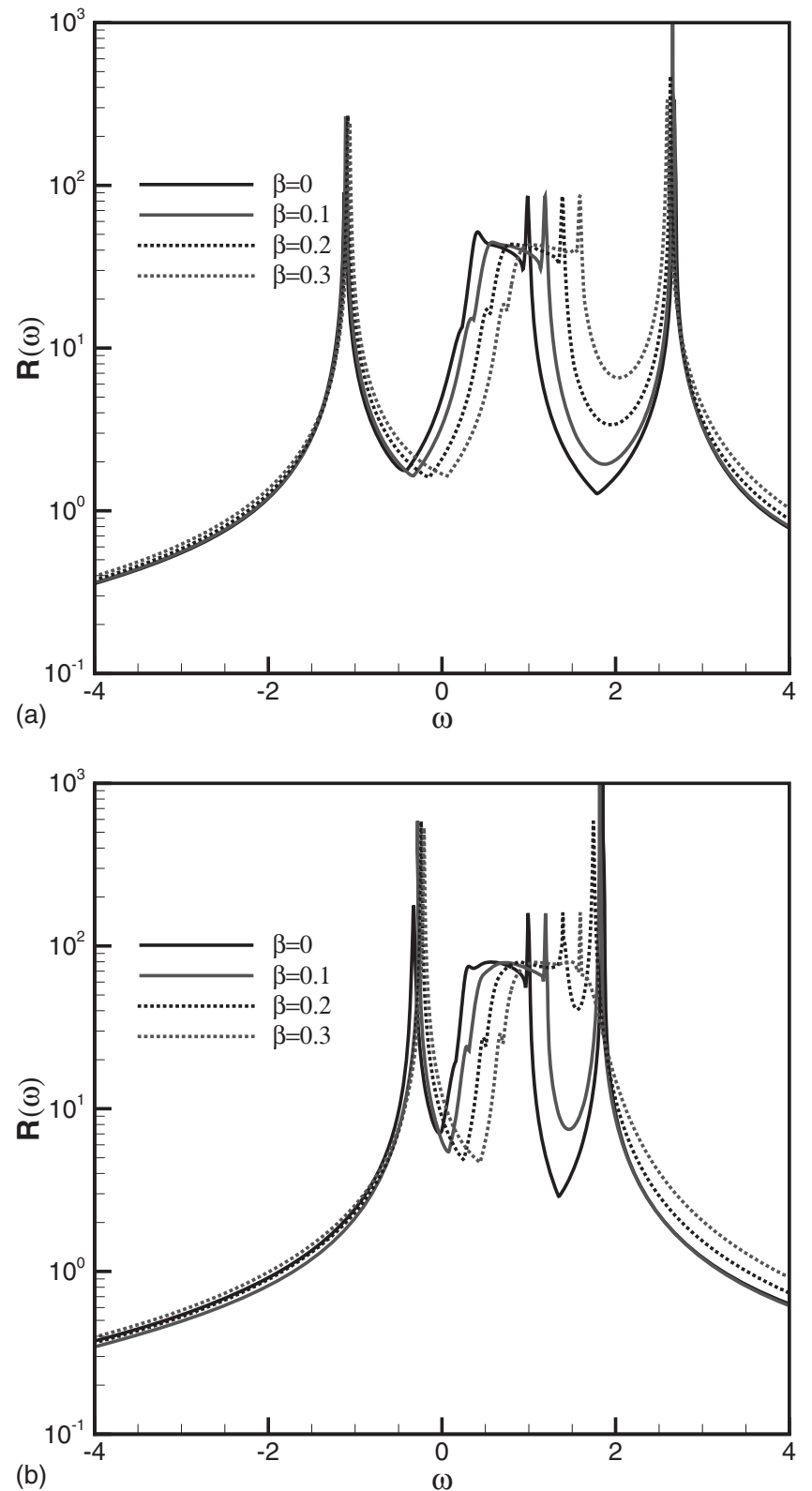

FIG. 10. Curves of the maximum response $R(\omega)$ to external excitation vs the frequency for the streamwise disturbance of $a=1, b=0$ with various $\beta$. (a) $\operatorname{Re}=3000$ and (b) $\operatorname{Re}=10000$. The other parameters are $\theta=0.5^{\prime}$ and $\zeta=0$.

bance and significantly increases with the increase of $\beta$ at a high Reynolds number. For spanwise disturbances, the initial growth rate of $G(t)$ is insensitive to $\beta$ for $\theta=1^{\circ}$.

In order to understand how $\beta$ influences the numerical abscissa, it is helpful to examine its effect on the behaviors of eigenvalues. In Figs. 4(a) and 5(a), with the increase of $\beta$, the Y-shaped branch and the surface mode move in the opposite directions. As a result, the distance between them significantly decreases with the increase of $\beta$. In Fig. 4(a), the surface mode is far from the Y-shaped branch, so the numerical abscissa is primarily determined by the modes of the Y-shaped branch. As to Fig. 5(a), because of the nonnormality between the surface mode and other modes, when the distance between the surface mode and the Y branch is small, the numerical abscissa significantly increases with the decrease of the distance. For the spanwise disturbances, as 

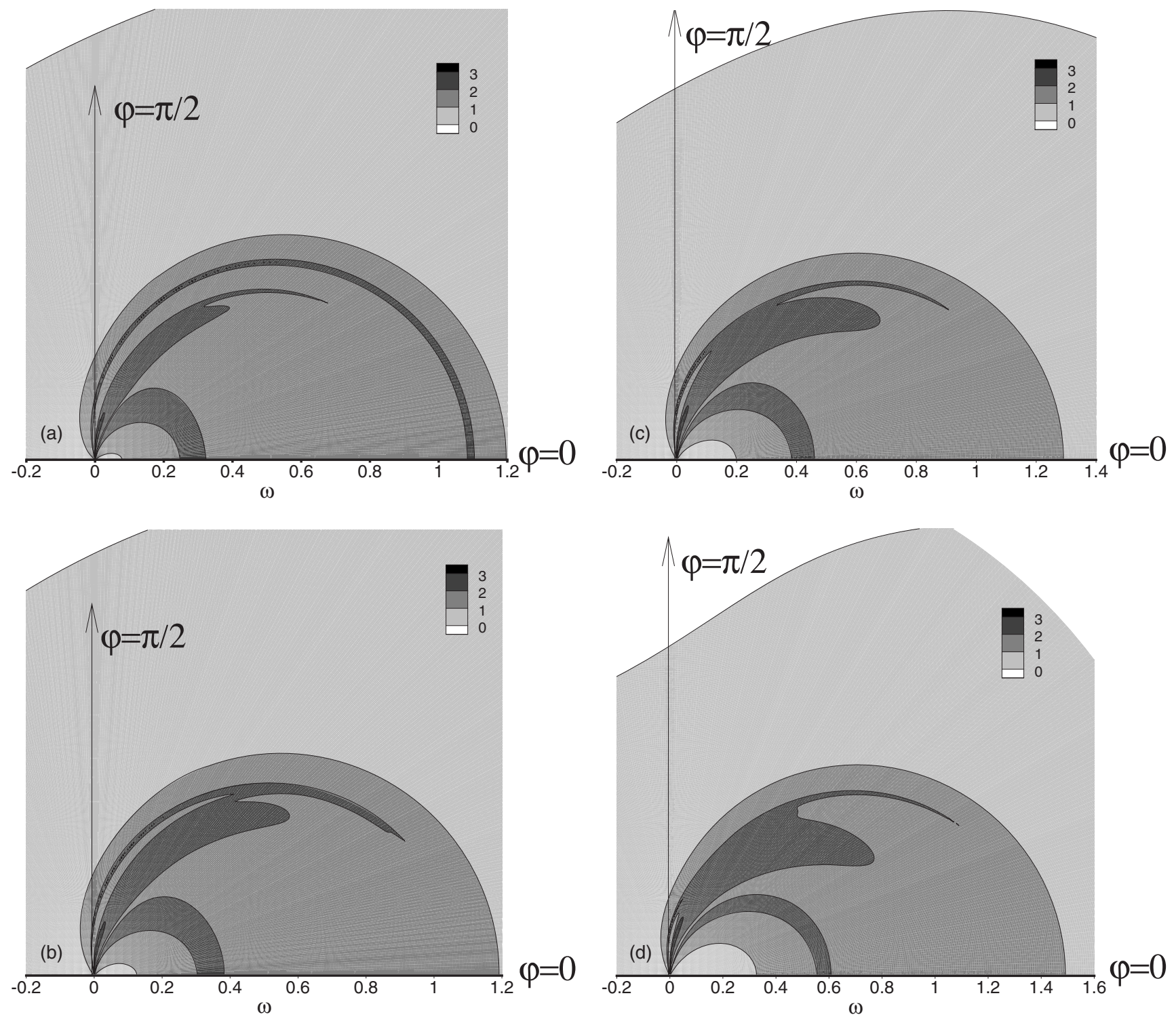

FIG. 11. The contours of the maximum responses $R(\omega)$ to external excitations in the $\omega$ - $\varphi$ plane with the wavenumber $k=1.0$. (a) $\beta=0$, (b) $\beta=0.05$, (c) $\beta$ $=0.1$, and $(\mathrm{d}) \beta=0.2$. The other parameters are $\theta=1^{\circ}, \zeta=0$, and $\operatorname{Re}=3000$.

shown in Figs. 4(b) and 5(b), the increase of $\beta$ destabilizes the Squire modes. However, with the increase of $\beta$ the distances between the surface modes and the Squire modes change little, so the numerical abscissa is insensitive to $\beta$ for spanwise disturbances.

Now we will examine the influence of $\beta$ for the case of $\theta=0.5^{\prime}$ for various Reynolds numbers. For spanwise disturbance, the structures of spectra of $\theta=0.5^{\prime}$ are qualitatively the same as that of $\theta=1^{\circ}$. So, we only present in Figs. 6(a)-6(c) the trajectories of eigenvalues of streamwise disturbances for $\theta=0.5^{\prime}$ as $\beta$ increases from 0 to 0.05 at $\operatorname{Re}$ $=1000,3000$, and 10 000. As shown in Fig. 6, the structures of the spectra of various Re are similar, i.e., there are two surface modes labeled $s$ in the $\omega$ plane. Comparing the structure of spectra with different Reynolds numbers, we find that with the increase of Re, the spectra become more continuous. As shown in Fig. 6, with the increase of $\beta$, the "Y"-shaped branch moves to the right; however, the two surface modes are insensitive to $\beta$. In each figure, it is apparent that the numerical abscissa increases with $\beta$. This result is qualitatively the same as that for $\theta=1^{\circ}$.

Comparing Figs. 6(a)-6(c), it is shown that with the increase of $\mathrm{Re}$, the two surface modes move toward the $\mathrm{Y}$ branch. We find it is surprising that the numerical abscissa decreases with the growth of the Reynolds number. This result means that the initial growth rate of $G(t)$ decreases with the increase of the Reynolds number. In most previous works, it is shown that the increase of the Reynolds number results in a larger transient growth. Here, we should note that the numerical abscissa only describes the trend of the transient growth at initial time; however, it does not mean that a larger numerical abscissa results in a larger maximum transient growth. 

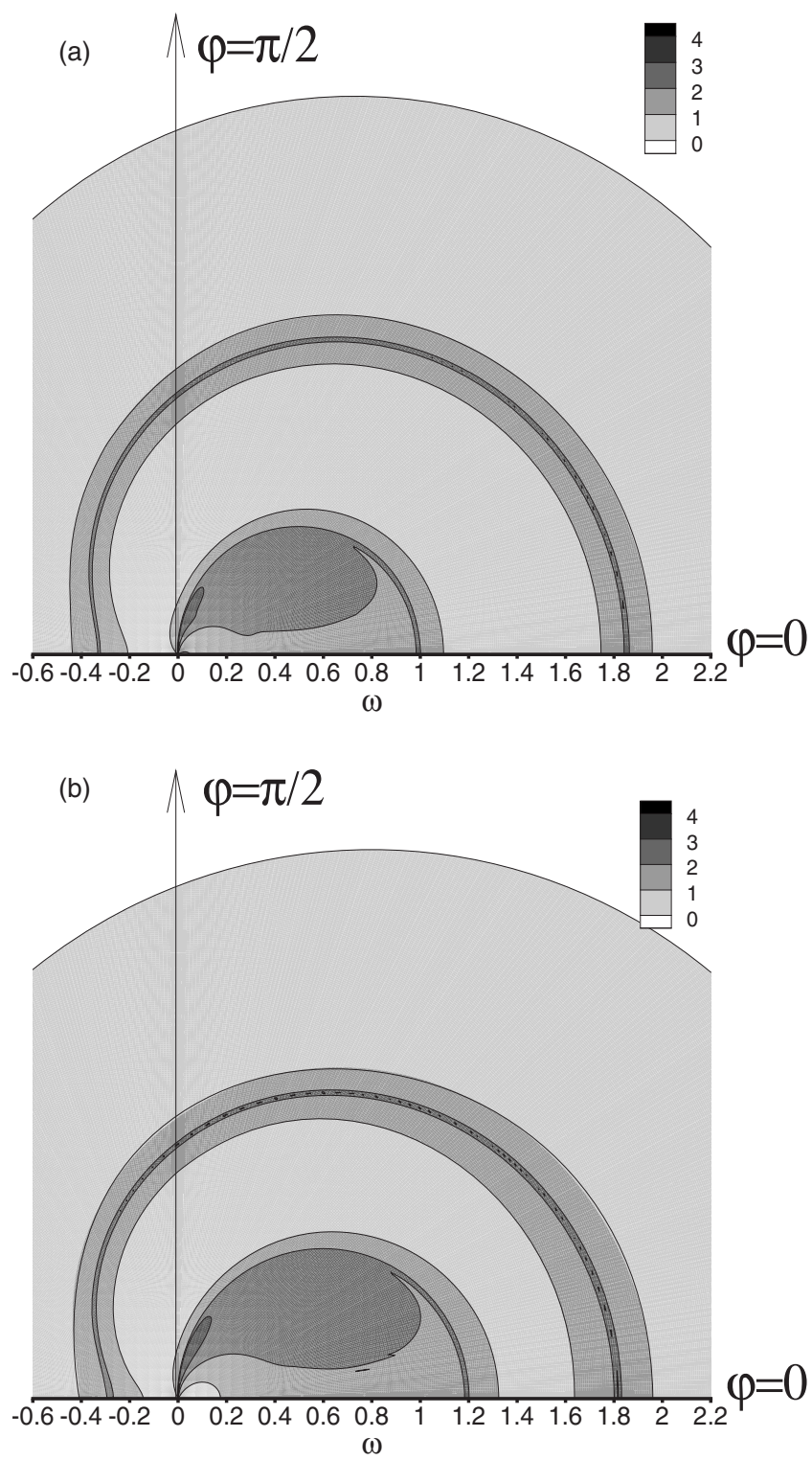
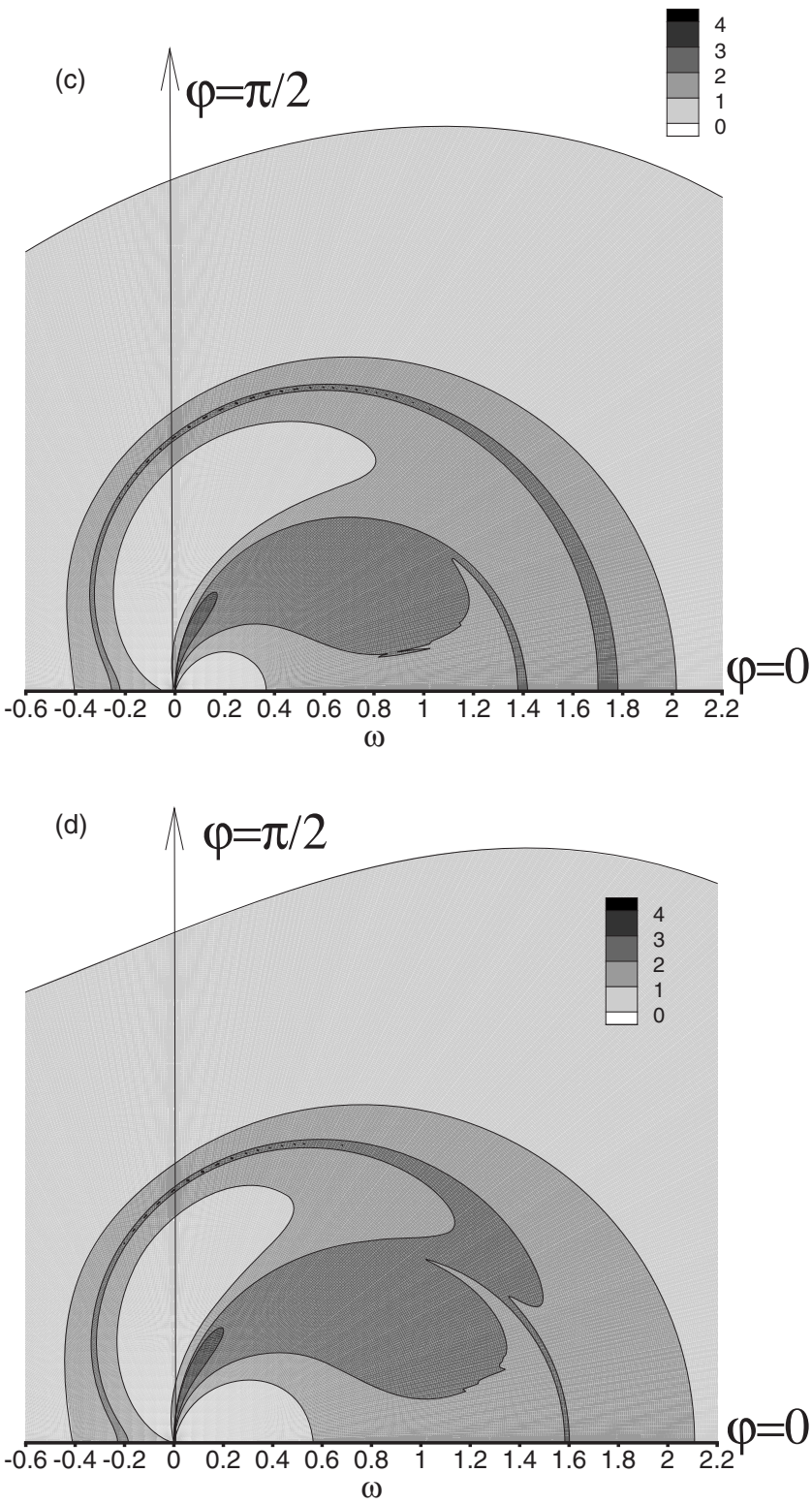

FIG. 12. The contours of the maximum responses $R(\omega)$ to external excitations in the $\omega$ - $\varphi$ plane with the wavenumber $k=1.0$. (a) $\beta=0$, (b) $\beta=0.1$, (c) $\beta$ $=0.2$, and (d) $\beta=0.3$. The other parameters are $\theta=0.5^{\prime}, \zeta=0$, and $\operatorname{Re}=10000$.

\section{Effect of porous medium on the response to external excitation}

As discussed in Sec. III, the resolvent norm represents the amplitudes of responses to excitations. So, the values of amplifications can be denoted by the level lines of pseudospectra.

Figure 7 displays the spectra and the pseudospectra for the streamwise disturbances for $\theta=1^{\circ}$ at $\operatorname{Re}=3000$. In these figures, at each point in the complex $\omega$ plane, the excitation is amplified by a factor of $\epsilon^{-1}$. In Fig. 7(a), for $\beta=0$, the middle branch is located in the region of $0.6 \leq \operatorname{Re}(\omega) \leq 0.7$. As shown in Figs. 7(a)-7(d), with the increase of $\beta$, the eigenvalues of the Y-shaped move to the right. As $\beta$ increases to 0.2 , the middle branch has moved in the region of $\operatorname{Re}(\omega)>1.0$. However, in these figures the surface mode maintains in the region near $\operatorname{Re}(\omega)=1$. For $\beta=0$, two relative maxima of the outermost contour appear in the $\omega$ plane. One is located near the left boundary of the $\mathrm{Y}$ branch, the other is near the surface mode. With the increase of $\beta$, the left maximum moves to the right and the right maximum maintains its original position. As $\beta$ increases to 0.2 , the surface mode is located directly above the middle branch and the two maxima coalesce and form a unique maximum. We also notice that with the increase of $\beta$, the numerical range protrudes more deeply into the unstable plane. So, the numerical abscissa increases with $\beta$. This result predicts that the increase of $\beta$ leads to a larger initial energy growth rate for streamwise disturbances.

Figure 8 displays the spectra, the pseudospectra, and the numerical range for $\theta=0.5^{\prime}$. As shown in Figs. 8(a) and 8(b), for $\mathrm{Re}=3000$, and Figs. 8(c) and 8(d) for $\mathrm{Re}=10$ 000, with the presence of the two surface modes the numerical range protrudes deeply into the unstable plane. This result indicates that these two surface modes are responsible for a large 

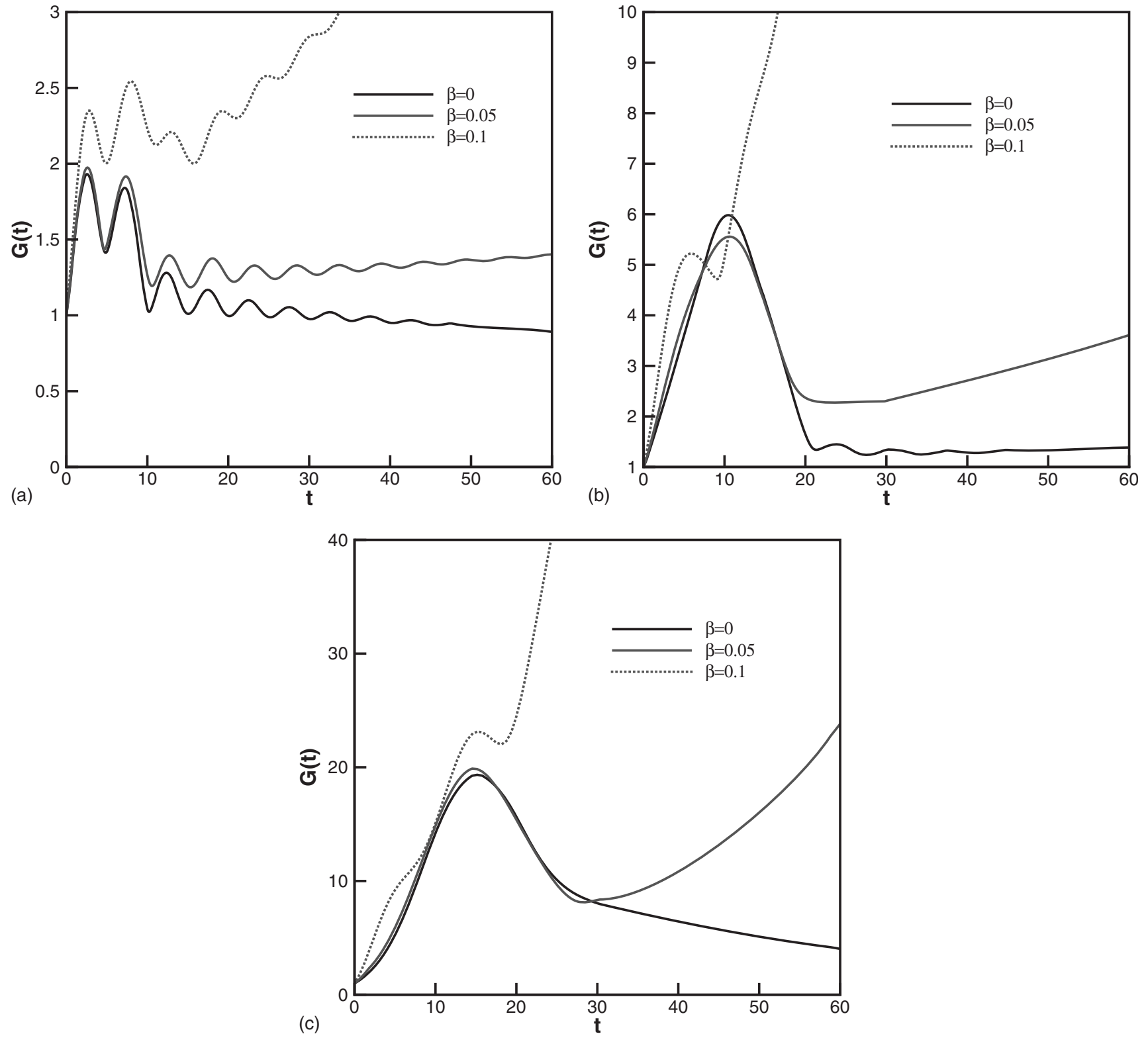

FIG. 13. Curves of the energy growth function $G(t)$ for streamwise disturbances with various $\beta$. (a) $\operatorname{Re}=300$, (b) $\operatorname{Re}=1000$, and (c) $\operatorname{Re}=3000$. The other parameters are $\theta=1^{\circ}, a=1, b=0$, and $\zeta=0$.

growth rate of $G(t)$ at initial time. Comparing Fig. 8(a) with Fig. 8(b), it is shown that the increase of $\beta$ from 0 to 0.05 results in a significant increase of the numerical abscissa. In Figs. 8(c) and 8(d), the influence of $\beta$ on the numerical abscissa at $\operatorname{Re}=10000$ is qualitatively the same as that in $\operatorname{Re}$ $=3000$. Comparing Fig. 8(a) with Figs. 8(c) and 8(d), it is observed that as the Reynolds number increases from 3000 to 10000 , both the left and the right surface modes move toward the Y branch. It seems that the numerical abscissa significantly decreases with the increase of the Reynolds number.

In the complex $\omega$ plane, we are particularly interested in the resonances of the real axis because it corresponds to external excitations at real frequencies. The maximal response $R(\omega)$ to external excitations can be defined as

$$
R(\omega)=\left\|(\omega \mathcal{I}-\mathcal{L})^{-1}\right\|_{E}, \quad \omega \in \mathbb{R} .
$$

In Fig. 9, the curves of the maximum resonances of streamwise disturbances versus the frequencies of external excitations are presented for $\theta=1^{\circ}$. In Fig. 9, each curve displays a multipeak structure. Each peak corresponds to the resonance of an eigenmode near the real $\omega$ axis. Comparing Fig. 9(a) with Fig. 4(a), it is found that the leftmost and the middle peaks correspond to the eigenmodes of the $\mathrm{Y}$ branch, and the rightmost peak corresponds to the surface mode. With the increase of $\beta$, the peaks corresponding to the $\mathrm{Y}$ branch move to the right and the rightmost peak moves to the left. For $\beta$ $=0.2$, when the peak excited by the surface mode collides with the middle peak, the rightmost peak significantly decreases. In Fig. 9(b), for $\mathrm{Re}=3000$, the curve of $\beta=0$ also 
has three peaks. For each curve, the left peak gradually decreases and moves to the right with the increase of $\beta$. As $\beta$ increases to 0.05 , the middle peak collides with the rightmost peak. As $\beta$ increases further, the peak generated by surface mode disappears and the middle peak becomes the rightmost peak. For the curves of $\theta=1^{\circ}$ in Fig. 9, we can conclude that when the surface mode is far from the Y branch, the surface mode could result in a large amplification of resonance. As the surface mode becomes closer to other modes of the $\mathrm{Y}$ branch, the response of surface mode is suppressed by other modes.

Figure 10 displays the curves of responses to external excitations for $\theta=0.5^{\prime}$. As shown in Fig. 6. for $\theta=0.5^{\prime}$, there are two surface modes located far from the $\mathrm{Y}$ branch. As shown in Figs. 10(a) and 10(b), the external excitations are significantly amplified in three regions, i.e., the leftmost peak, the middle roof, and the rightmost peak. The leftmost and the rightmost peaks are generated by the two surface modes, and the middle roof is generated by the $\mathrm{Y}$ branch. With the increase of $\beta$, the middle roof moves to the right. In Fig. 10(a), for $\operatorname{Re}=3000$, because the middle roof is far from the right peak, the shape of the roof is almost unchanged. However, when $\beta$ moves to the right, the left peak gets higher and the right peak gets lower. In Fig. 10(b), for Re $=10000$, as $\beta$ increases to 0.3 the middle roof has collided with the rightmost peak and the peak corresponding to the right surface mode disappears. This result indicates that the shear modes could reduce the response of surface mode. In the present paper, we only present the response of streamwise disturbances. For spanwise disturbances, the structures of the curves of responses versus frequency are similar for different $\beta$.

In the preceding part we have studied the resonances of streamwise disturbances. In order to know more about the physics of the responses to external excitations, we will examine the responses of three-dimensional disturbances to excitations of real frequencies. We define the propagating angle as $\varphi=\arctan (b / a)$. When studying the problem, we will fix the magnitude of the wavenumber and other parameters and only change the angle $\varphi$.

In Figs. 11 and 12, we plot the level lines of $\log [\mathrm{R}(\omega)]$ in polar coordinates in which $\omega$ is the radius and $\varphi$ is the azimuth. $\varphi=0$ and $\pi / 2$ correspond to the streamwise and the spanwise disturbances, respectively. In Fig. 11, for $\theta=1^{\circ}$ at $\operatorname{Re}=3000$, the maximum response occurs in the spanwise direction at the vicinity of $\omega=0$. In the spanwise direction, for a large $\omega$ the response is dramatically reduced. This result indicates that the spanwise disturbances are only sensitive to low frequency excitations for $\theta=1^{\circ}$. As shown in Fig. 11, the structures of the responses with different $\beta$ are qualitatively similar. In Figs. 11(a)-11(d), it is found that the perturbations in the shaded semicircle areas are significantly amplified. It is interesting that the semicircle areas are mainly located in the regions of $\varphi<\pi / 2$. This result predicts that for a given $\omega$ the disturbances are mainly amplified downstream, and the responses of upstream disturbances are weaker for $\theta=1^{\circ}$ at $\operatorname{Re}=3000$.
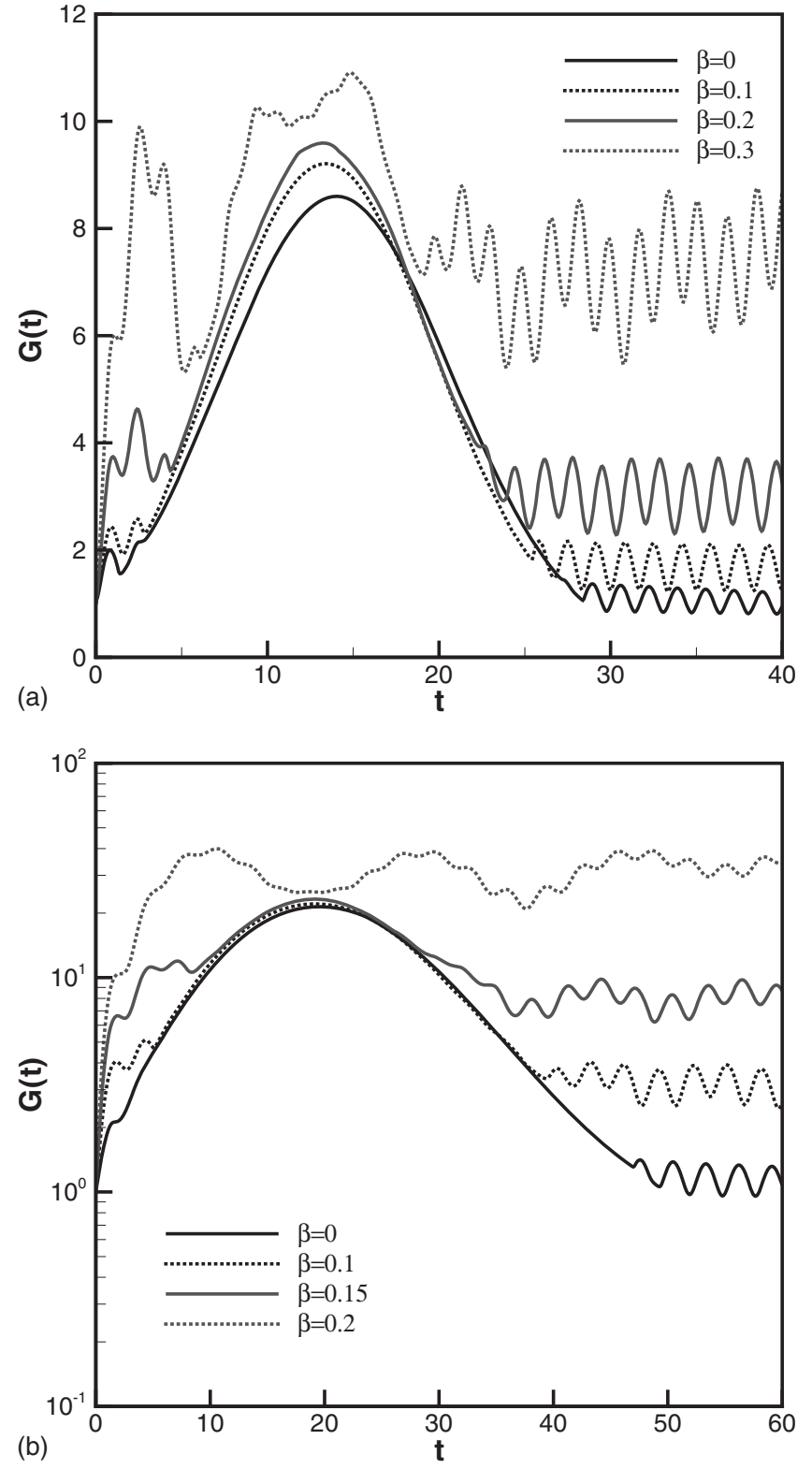

FIG. 14. Curves of the energy growth function $G(t)$ for streamwise disturbances with various $\beta$. (a) $\operatorname{Re}=3000$ and (b) $\operatorname{Re}=10000$. The other parameters are $\theta=0.5^{\prime}, a=1, b=0$, and $\zeta=0$.

In Fig. 12, the level lines of responses are plotted for $\theta$ $=0.5^{\prime}$ at $\operatorname{Re}=10$ 000. As shown in Fig. 12(a), with the presence of the two surface modes, a semicircular strip is present in the $\omega-\varphi$ plane. The two surface modes play an important role in generating this semicircle strip, and the inner semicircular area is primarily generated by the eigenmodes of the $\mathrm{Y}$ branch. In Figs. 12(a)-12(d), with the increase of $\beta$, the inner semicircular area extends to the right. In Figs. 12(a) and 12(b), the semicircular strip maintains in its original place. As $\beta$ increases to 0.2 , the inner semicircular area collides with the outer strip. As shown in Figs. 12(c) and 12(d), the region of large amplification is in a form of a semicircle with $\omega$ extending in the region of $-0.4-2.0$. Comparing Fig. 12 with Fig. 11, we found that for $\beta=0.5^{\prime}$ external excitations can be amplified upstream. 

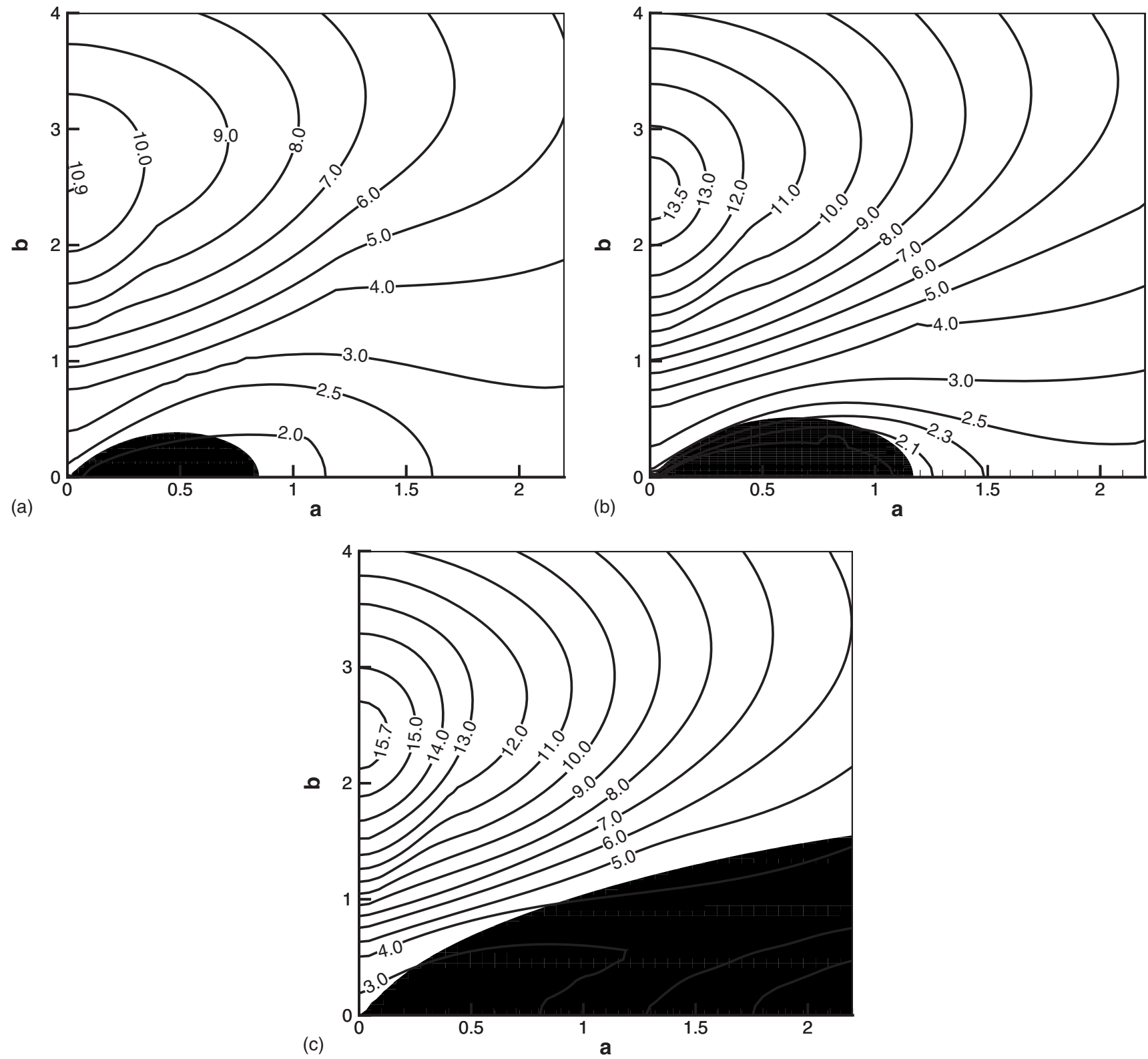

FIG. 15. Level lines of the maximum energy growth $G_{\max }$ for $\operatorname{Re}=300$. (a) $\beta=0$, (b) $\beta=0.05$, and (c) $\beta=0.1$. The shaded areas are the linear unstable regions. The other parameters are $\theta=1^{\circ}$ and $\zeta=0$.

\section{Effect of porous medium on the optimal energy growth}

We will examine the responses of the streamwise disturbances to the initial conditions. As discussed in Sec. III, the optimal energy growth at time $t$ is given by the energy growth function $G(t)$. In Figs. 13 and 14, we plot the curves of the energy growth function of streamwise disturbances for $\theta=1^{\circ}$ and $0.5^{\prime}$ at various Reynolds numbers. In Fig. 13(a), for $\mathrm{Re}=300$, oscillatory behaviors of the energy growth are observed. We note that the initial growth rates of different $\beta$ are almost indistinguishable. This result is consistent with that the increase of $\beta$ has little influence on the numerical abscissa in Fig. 4(a). In Fig. 13(a), for $\beta=0$, the oscillatory transient is followed by a damped stage. As $\beta$ increases to 0.1 , after the oscillatory transient stage $G(t)$ increases with time.
In Figs. 13(b) and 13(c), for larger Reynolds numbers $\operatorname{Re}=1000$ and 3000 , the oscillatory behaviors are not apparent. This result indicates that the effect of the surface mode on the transient energy growth is suppressed by other modes. In these two figures, it is shown that the initial growth increases with $\beta$. The parameters in Fig. 13(c) are the same as that in Fig. 5(a). In Fig. 5(a), the numerical abscissa apparently increases with $\beta$. From the above discussions on Fig. 13 , we infer that if at the initial stage the energy growth is primarily generated by the surface mode, the abscissa is insensitive to $\beta$; if the energy growth is generated by the shear modes, the initial growth increases with $\beta$.

Figures 14(a) and 14(b) plot the growth function of streamwise disturbances at $\mathrm{Re}=3000$ and 10000 for $\theta$ $=0.5^{\prime}$. In Fig. 14(a), for $\beta=0,0.1,0.2$, the peak in the transient phase is approximately located in the range of $10 \leq t$ 

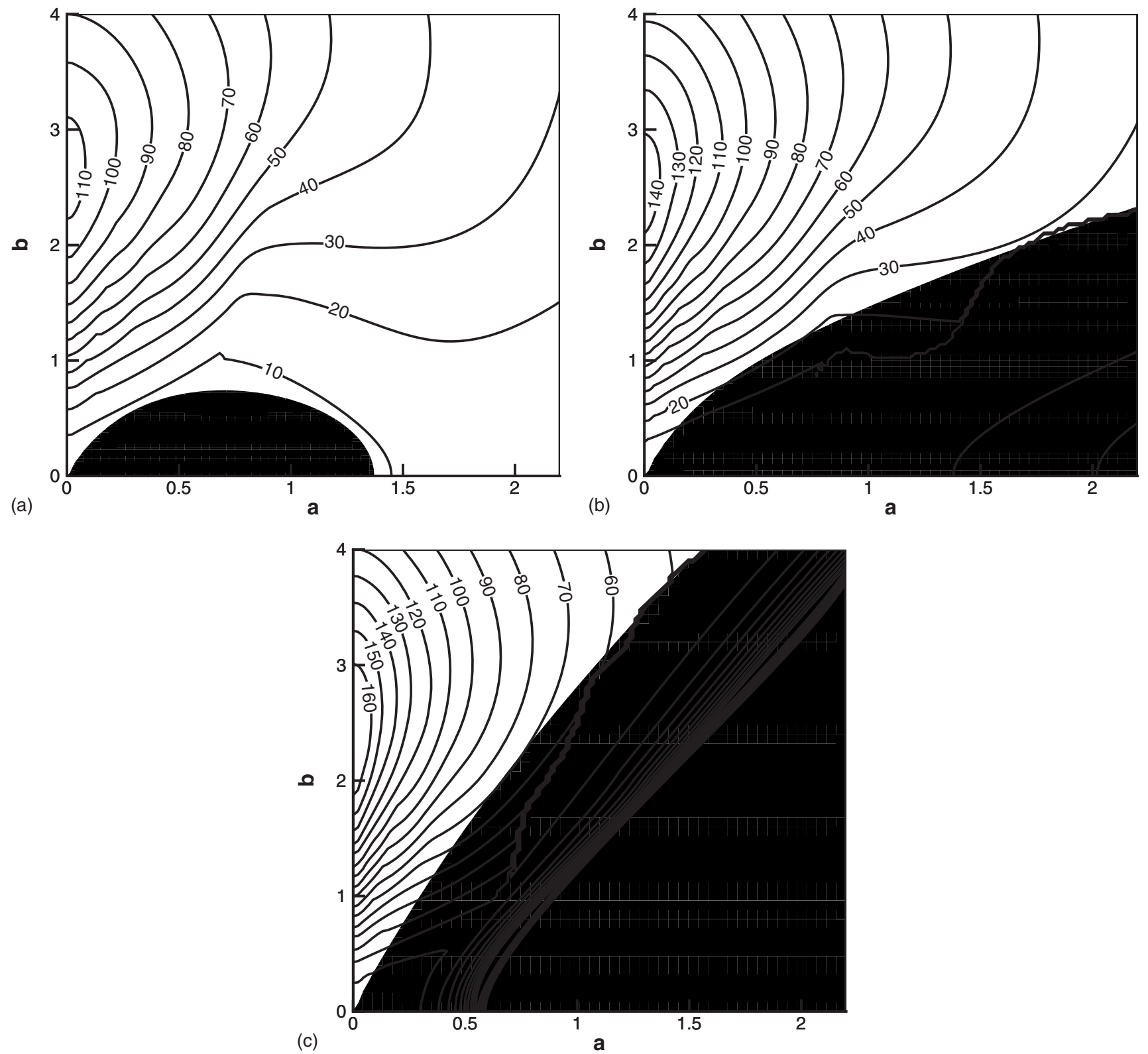

FIG. 16. Level lines of the maximum energy growth $G_{\max }$ for $\operatorname{Re}=1000$. (a) $\beta=0$, (b) $\beta=0.05$, and (c) $\beta=0.1$. The shaded areas are the linear unstable regions. The other parameters are $\theta=1^{\circ}$ and $\zeta=0$.

$\leq 15$. Oscillatory behaviors are observed in the initial stage and after the transient phase. The parameters in Fig. 14(a) are the same as that in Figs. 8(a) and 8(b). With the increase of $\beta$, in Figs. 8(a) and 8(b) the numerical range protrudes more deeply into the unstable half plane. As shown in Fig. 14(a), it is apparent that the initial growth rate increases with $\beta$.

In Fig. 14(b), for a larger Reynolds number $\mathrm{Re}=10$ 000, the peak of the transient growth is located at $t \approx 20$. Being similar to Fig. 14(a) in Fig. 14(b), the peak in the transient stage is primarily generated by shear mode. Oscillatory behaviors generated by surface modes also present in this figure. Being different to Fig. 13 for $\theta=1^{\circ}$, it seem that for $\theta$ $=0.5^{\prime}$, as the Reynolds number increases from 3000 to 10000 , the oscillatory behaviors have not been completely suppressed by the shear modes. In Fig. 14(b), the increase of $\beta$ results in a larger initial growth rate; however, the peak of the transient growth is insensitive to $\beta$. As $\beta$ increases to 0.2 , the peak of the transient growth at $t \approx 20$ is dominated by oscillatory peaks. In this case, the transient growth generated by shear modes is switched to oscillatory behaviors.

We have examined the optimal growth for the streamwise disturbances. In order to know the characteristics of transient growth of three-dimensional disturbances, we will compute the maximum energy growth $G_{\max }$ in the $a-b$ plane. Here, $G_{\max }=\max [G(t)]$. Figure 15 plots the level curves of $G_{\max }$ at $\operatorname{Re}=300$ for $\theta=1^{\circ}$. In Fig. 15(a) for $\beta=0$, a small unstable area is revealed in the $a-b$ plane. In this area, the surface mode is linearly unstable. As shown in Fig. 15(a), the unstable area is confined in the range of $a<1$. With the increase of $\beta$, as shown in Figs. 15(b) and 15(c), the unstable area significantly extends in the $a-b$ plane. In Figs. 14(a)-14(c), we find that the spanwise disturbances have the 

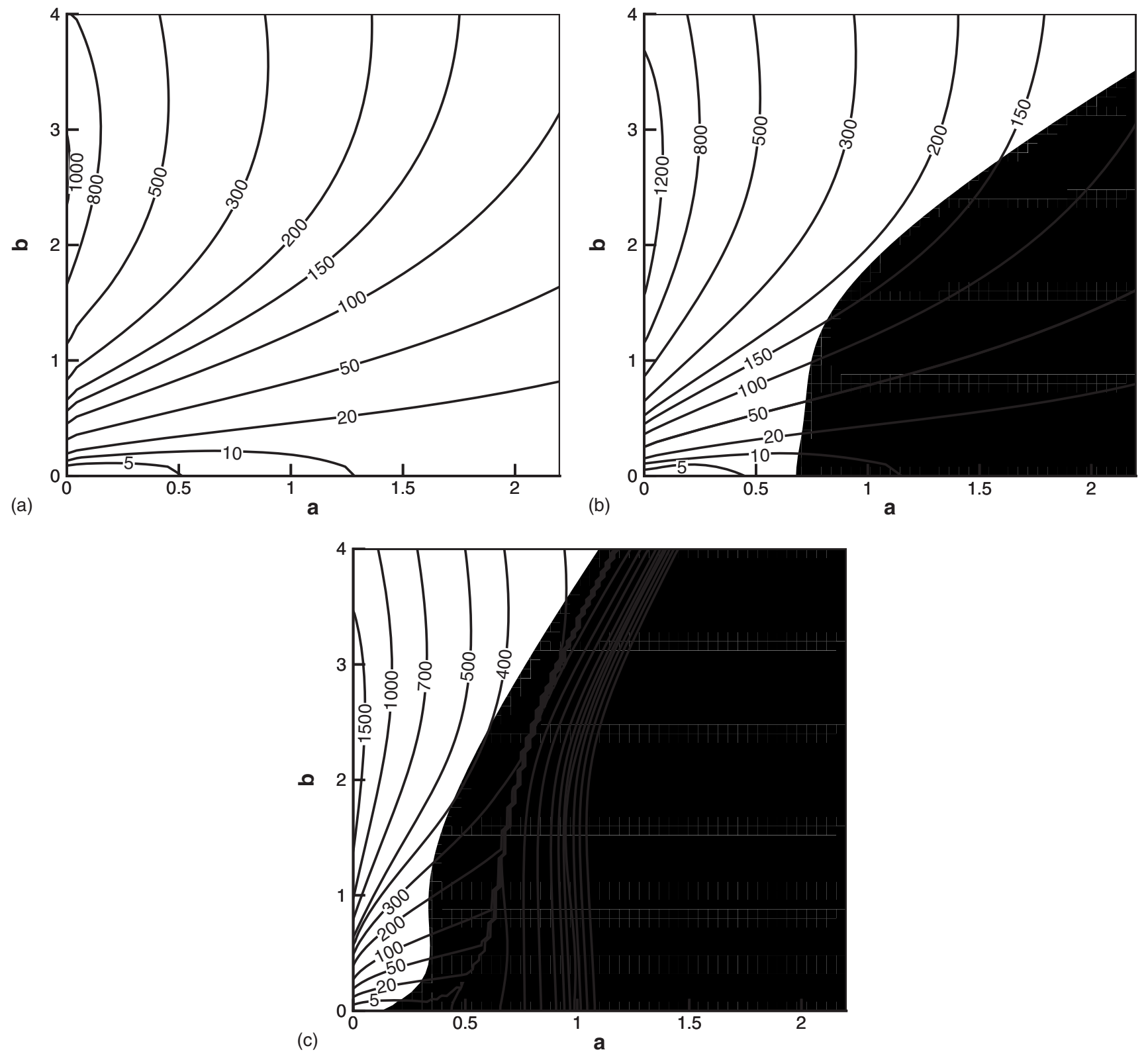

FIG. 17. Level lines of the maximum energy growth $G_{\max }$ for $\operatorname{Re}=3000$. (a) $\beta=0$, (b) $\beta=0.1$, and (c) $\beta=0.2$. The shaded areas are the linear unstable regions. The other parameters are $\theta=0.5^{\prime}$ and $\zeta=0$.

maximum transient growth; however, the spanwise disturbances are always linear stable. For $\beta=0,0.05,0.1$, the maximum transient growth are about $10.9,13.5$, and 15.7 , respectively.

Figure 16 represents $G_{\max }$ in the $a-b$ plane at $\operatorname{Re}=1000$ for $\theta=1^{\circ}$. The structures of the level curves are qualitatively similar to that in Fig. 15. The spanwise disturbances have the maximum transient growth. The maximum transient growth are about 110,140 , and 160 for $\beta=0,0.05,0.1$, respectively. In Figs. 15 and 16, it is shown that the maximum transient growth increases with $\beta$. Comparing Fig. 15 with Fig. 16, it is shown that with the increase of Re, the maximum energy growth increases, and the unstable regions significantly extend in the $a-b$ plane.

Figure 17 presents the level lines of $G_{\max }$ in the $a-b$ plane for small inclined angle of $\theta=0.5^{\prime}$ at $\mathrm{Re}=3000$. The marginal curves for $\theta=0.5^{\prime}$ at $\operatorname{Re}=3000$ are presented in Fig. 3. For $\beta=0$ and $\operatorname{Re}=3000$, the system is linearly stable. So, in Fig. 17(a), for $\beta=0$, there is no unstable region. With the increase of $\beta$, the surface mode becomes more unstable. However, we should note that for $\theta=0.5^{\prime}$ the most unstable surface mode is not in the long-wave range. In Fig. 17(b), for $\beta=0.1$, an unstable region is found in the region of $a>0.5$. As $\beta$ increases to 0.2 , the unstable region significantly expands in the $a-b$ plane. Comparing Fig. 17(b) with Fig. $17(\mathrm{c})$, it is apparent that with the increase of $\beta$, the unstable region extends toward the long-wave region. Being different to Figs. 15 and 16 for $\theta=1^{\circ}$, in Fig. 17, the unstable regions do not cover the vicinity of the origin of the $a-b$ plane. Being similar to Figs. 15 and 16, the spanwise disturbances have the maximum growth, and the maximum growth increases with $\beta$. 


\section{CONCLUSIONS}

In the present paper, the problem of a liquid film flowing down a porous layer is studied in the framework of the nonmodal stability theory. A one-sided model is used to describe the flow of the film. The influence of the porous medium on the fluid layer reduces to a slip of velocity at the fluid-porous interface and can be identified by a parameter $\beta$. This parameter influences the distribution of the base velocity and the boundary conditions. Our main focus has been to investigate the effect of porous medium on the linear stabilities of the eigenmodes and the transient behaviors of a liquid film flowing down a porous plane.

We have studied the linear stability of streamwise disturbances for two typical inclined angles of $\theta=1^{\circ}$ and $0.5^{\prime}$. The marginal curves of the two-dimensional disturbances display a bimodal structure. The parameter $\beta$ plays a crucial role in determining the stabilities of the system. With the increase of $\beta$, the marginal curve of surface mode becomes more unstable and the curve of shear mode becomes more stable.

In order to gain more insight into the physics of the influence of the porous medium on the stability, we examine the trajectories of the eigenvalues for various $\beta$. For $\theta=1^{\circ}$, the spectra of the streamwise disturbances include a surface mode and a series of discrete eigenmodes in the form of a Y-shaped structure. For $\theta=0.5^{\prime}$, two surface modes are revealed, one propagates downstream and the other propagates upstream. With the increase of $\beta$, the eigenvalues in the $\mathrm{Y}$ branch move to the right in the $\omega$ plane, and the surface mode maintains its original position. For lower Reynolds numbers, the increase of $\beta$ has little influence on the numerical range and the numerical abscissa. For larger Reynolds numbers, the increase of $\beta$ significantly increases the value of numerical abscissa.

The pseudospectra and the energy growth function are studied in the framework of the nonmodal stability theory. We focus on the effect of $\beta$ on the responses to external excitations and the responses to initial conditions. Our results show that the spanwise disturbances have the maximum response at $\omega=0$. The curves of responses to the streamwise excitations display multipeak structures. These peaks correspond to the responses to the surface modes and the shear modes. With the increase of $\beta$, the peaks of the shear modes move to the right; however, the position of the peaks corresponding to the surface modes change little with $\beta$. When the peaks of the shear modes and the surface modes are close, the responses of the surface modes significantly decrease. We also examine the response of the three-dimensional disturbances to excitations. It is shown that for $\theta=1^{\circ}$, the downstream responses are the dominant, and for $\theta=0.5^{\prime}$ the upstream surface mode can also be significantly amplified.

The characteristics of transient energy growth are studied for $\theta=1^{\circ}$ and $0.5^{\prime}$. For $\theta=1^{\circ}$, the presence of the surface mode results in oscillatory behavior at the initial stage or after the transient stage. For a lower Reynolds number, the initial growth rate of $G(t)$ is insensitive to $\beta$. For a larger Reynolds number, the initial growth rate of $G(t)$ increases with $\beta$. For $\theta=1^{\circ}$, the results show that the increase of the Reynolds number suppresses the oscillatory behavior in- duced by the surface mode. For $\theta=0.5^{\prime}$, there are two surface modes contributing to the transient growth. With the increase of $\beta$, the maximum of $G(t)$ increases slightly, but the initial growth rate significantly increases. We have found that the increase of the Reynolds number does not completely suppress the oscillatory behavior at $\theta=0.5^{\prime}$.

We have plotted the level lines for the optimal energy growth of the three-dimensional disturbances. For $\theta=1^{\circ}$, an unstable area is revealed in the $a-b$ plane. With the increase of $\beta$, the unstable region extends in the $a-b$ plane. For $\theta$ $=0.5^{\prime}$, the structures of the level lines are similar to that of $\theta=1^{\circ}$. Only when $\beta$ exceeds a certain value, the unstable region begin to present in the $a-b$ plane. For both $\theta=1^{\circ}$ and $0.5^{\prime}$, it is shown that the spanwise disturbances have the maximum transient growth and this maximum increases with $\beta$.

The results of the nonmodal stability theory show that the increase of $\beta$ results in a larger transient energy growth. This conclusion is counterintuitive, given the result of the eigenvalue analysis that with the increase of $\beta$ the marginal curve of the shear mode becomes more stable.

\section{ACKNOWLEDGMENTS}

The authors thank the financial support for this research from the National Foundation of China (Grant Nos. 10772185 and 50890182) and the Knowledge Innovation Program of Chinese Academy of Sciences (Contract No. KGCX-SW-409).

${ }^{1}$ T. B. Benjamin, "Wave formation in laminar flow down an inclined plate," J. Fluid Mech. 2, 554 (1957).

${ }^{2}$ C. S. Yih, "Stability of liquid flow down an inclined plane," Phys. Fluids 6, 321 (1963).

${ }^{3}$ G. J. De Bruin, "Stability of a layer of liquid flowing down an inclined plane," J. Eng. Math. 8, 259 (1974).

${ }^{4}$ S. P. Lin, "Instability of a liquid film flowing down an inclined plane," Phys. Fluids 10, 308 (1967).

${ }^{5}$ J. M. Floryan, S. H. Davis, and R. E. Kelly, "Instabilities of a liquid film flowing down a slightly inclined plane," Phys. Fluids 30, 983 (1987).

${ }^{6}$ J. P. Pascal, "Linear stability of fluid flow down a porous inclined plane," J. Phys. D 32, 417 (1999).

${ }^{7}$ G. S. Beavers and D. D. Joseph, "Boundary conditions at a naturally permeable wall," J. Fluid Mech. 30, 197 (1967).

${ }^{8}$ J. P. Pascal, "Instability of power-law fluid flow down a porous incline," J. Non-Newtonian Fluid Mech. 133, 109 (2006).

${ }^{9}$ I. M. R. Sadiq and R. Usha, "Thin Newtonian film flow down a porous inclined plane: Stability analysis," Phys. Fluids 20, 022105 (2008).

${ }^{10}$ R. Liu and Q. S. Liu, "Instabilities of a liquid film flowing down an inclined porous plane," Phys. Rev. E 80, 036316 (2009).

${ }^{11}$ L. N. Trefethen and M. Embree, Spectra and Pseudospectra: The Behavior of Nonnormal Matrices and Operators (Princeton University Press, Princeton, NJ, 2005).

${ }^{12}$ P. J. Schmid and D. S. Henningson, Stability and Transition in Shear Flows (Springer, New York, 2001).

${ }^{13}$ P. J. Olsson and D. S. Henningson, "Optimal disturbance growth in watertable flow," Stud. Appl. Math. 94, 183 (1995).

${ }^{14} \mathrm{M}$. A. Combarnous and S. A. Bories, "Hydrothermal convection in saturated porous media," Adv. Hydrosci. 10, 231 (1975)

${ }^{15}$ P. J. Schmid, "Nonmodal stability theory," Annu. Rev. Fluid Mech. 39, 129 (2007)

${ }^{16}$ S. C. Reddy and D. S. Henningson, "Energy growth in viscous channel flows," J. Fluid Mech. 252, 209 (1993).

${ }^{17}$ S. A. Orszag, "Accurate solution of the Orr-Sommerfeld stability equation,” J. Fluid Mech. 50, 689 (1971). 
${ }^{18}$ C. Canuto, M. Y. Hussaini, A. Quarteroni, and T. A. Zang, Spectral Methods in Fluid Dynamics (Springer-Verlag, New York, 1993).

${ }^{19}$ M. H. Chang, F. Chen, and B. Straughan, "Instability of Poiseuille flow in a fluid overlying a porous layer," J. Fluid Mech. 564, 287 (2006).
${ }^{20}$ R. Liu, Q. S. Liu, and S. C. Zhao, "Instability of plane Poiseuille flow in a fluid-porous system," Phys. Fluids 20, 104105 (2008).

${ }^{21}$ L. N. Trefethen, Spectral Methods in MATLAB (SIAM, Philadelphia, 2000). 